\title{
Conductance distribution in 1D systems: dependence on the Fermi level and the ideal leads
}

\author{
I. M. Suslov \\ P.L.Kapitza Institute for Physical Problems, \\ 119334 Moscow, Russia \\ E-mail: suslov@kapitza.ras.ru
}

\begin{abstract}
The correct definition of the conductance of finite systems implies a connection to the system of the massive ideal leads. Influence of the latter on the properties of the system appears to be rather essential and is studied below on the simplest example of the $1 \mathrm{D}$ case. In the lognormal regime this influence is reduced to the change of the absolute scale of conductance, but generally changes the whole distribution function. Under the change of the system length $L$, its resistance may undergo the periodic or aperiodic oscillations. Variation of the Fermi level induces qualitative changes in the conductance distribution, resembling the smoothed Anderson transition.
\end{abstract}

\section{Introduction and main results}

The correct definition of the conductance of finite systems is not a trivial issue, which was a subject of the vivid discussion at early 1980-ties [1- 9] (see the review article [10]). The reason of controversy was related to a fact that the conductance of finite systems is an ill-defined quantity. It is a consequence of the specific feature of the linear response formulas: the $\delta$-functions, contained in them, should be extended to the width $\Gamma$, which should be tended to zero only after the thermodynamic limit transition; such procedure is surely impossible in finite systems. To avoid this difficulty, the rather elegant trick was suggested 3: the finite system is connected to the ideal leads (Fig.1), which are suggested to be sufficiently massive, so the thermodynamic limit is practically taken in these leads. Such construction solves the problem of interpretation of the Kubo formula but creates new problems: the corresponding definition of conductance refers to the composite system "sample+ideal leads", and its relation to the initial system remains the open question. In order to clarify a situation, one can introduce the semi-transparent interfaces between the system and the ideal leads [11. Influence of the latter on the properties of the system is surely essential near the Anderson transition [12, but was discussed in 11, 12, only on the abstract level. In fact, this influence can be a subject of the constructive analysis, which is demonstrated below on the simplest example of 1D systems.

In theoretical papers, one usually suggests the ex- istence of the unique Fermi level, while a difference between the sample and ideal leads is determined by the absence of the random potential in the latter case (Fig.2,a). Practically, such situation is not very realistic: usually, in the experimental device the connecting leads are produced from a good metal with the large Fermi energy (so impurities are effectively screened), while the typical disordered system is a semi-metal or a doped semiconductor, where effects of disorder are strikingly manifested (Fig.2,b). Nevertheless, the difference from the former case (Fig.2,a) does not look essential and is usually ignored in theoretical papers. However, the use of the foreign leads becomes inevitable, if the Fermi level in the system corresponds to the forbidden band of the ideal crystal (Fig.2,c), where allowed states arise only due to a random potential. In this case, the attempt to make the connecting leads from the same material without impurities will end by a confusion: "the ideal leads" will not conduct at all.

An explicit introduction of the ideal leads (Fig.2, b, c) is realized with a help of the edge transfer matrices (Sec.2) and sets the interesting physical problem: producing small variation of the Fermi level (not essential for the ideal leads), one can transfer from the quasi-metallic regime in the allowed band (Fig.2,b) to the fluctuational states in the deep of the forbidden band (Fig.2,c). In the course of it, the conductance distribution of the 1D system undergoes the qualitative changes resembling the smoothed Anderson transition. 


\begin{tabular}{l|l|l}
\hline $\begin{array}{l}\text { ideal } \\
\text { conductor }\end{array}$ & $\begin{array}{l}\text { ideal } \\
\text { conductor }\end{array}$ \\
\hline
\end{tabular}

Figure 1: The correct definition of the conductance of finite systems implies a connection of the massive ideal leads. For the discussion of influence of the latter, one can introduce the semi-transparent boundaries 11.

If $g$ is the dimensionless conductance (conductance $G$ of the system of size $L$ in quantum units $\left.e^{2} / h\right)$, then the distribution $P(\rho)$ of dimensionless resistance $\rho=1 / g$ is described by the following evolution equation

$$
\frac{\partial P(\rho)}{\partial L}=\alpha \frac{\partial}{\partial \rho}\left[\rho(1+\rho) \frac{\partial P(\rho)}{\partial \rho}\right]
$$

derived in the number of papers [13]-18] and considered as sufficiently universal. However, more general equation was suggested in [12] for 1D systems

$$
\frac{\partial P(\rho)}{\partial L}=\tilde{\alpha} \frac{\partial}{\partial \rho}\left[-\gamma(1+2 \rho) P(\rho)+\rho(1+\rho) \frac{\partial P(\rho)}{\partial \rho}\right],
$$

which is reduced to (1) in the random phase approximation. The latter approximation is sufficiently good for the deep of the allowed band and the "natural" ideal leads (Fig.2,a), which is usually suggested in theoretical papers (see references in [19, 20]), while the situation in the forbidden band is considered infrequently [21, 22, 23. and only on the level of wave functions. The main argument of the paper 12] was based on the fact, that a finite value of the parameter $\gamma$ arises in the case of semi-transparent boundaries (Fig.1), even if the random phase approximation is applicable to the system under consideration.

Still more general evolution equation arises for the explicitly introduced ideal leads (Sec.7)

$$
\begin{aligned}
\frac{\partial P(\rho)}{\partial L} & =\tilde{\alpha} \frac{\partial}{\partial \rho}\left[-\gamma_{1}(1+2 \rho) P(\rho)-\right. \\
& \left.-2 \gamma_{2} \sqrt{\rho(1+\rho)} P(\rho)+\rho(1+\rho) \frac{\partial P(\rho)}{\partial \rho}\right],
\end{aligned}
$$

which is reduced to (2) in the regions of small and large $L$, where the typical values of $\rho$ are small and large correspondingly (then $\gamma=\gamma_{1}$ in the first case and $\gamma=\gamma_{1}+\gamma_{2}$ in the second one), so equation (3) is analogous to equation (2) with variable $\gamma$. A possibility of the $\gamma$ variation in the course of evolution
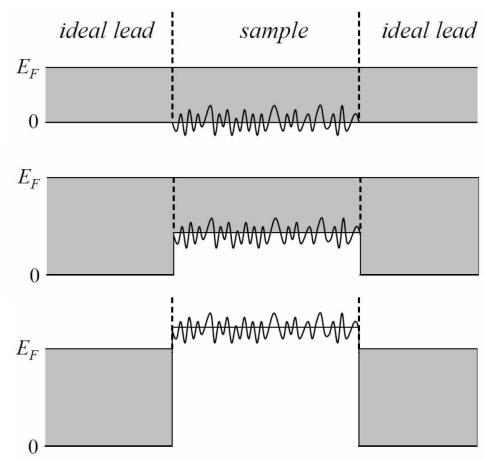

(b)

Figure 2: (a) In the theoretical papers one usually suggests that a difference between the sample and ideal leads is determined by the absence of a random potential in the latter case; (b) Practically in the experiment the connecting leads are produced from a good metal with the large Fermi energy; (c) Explicit introduction of the ideal leads is inevitable, if the Fermi level corresponds to the forbidden band of the ideal crystal.

was allowed in [12 from the very beginning and is systematically studied in Sec.3. The limiting value of $\gamma$ for large $L$ is determined by internal properties of the system under consideration and does not depend on ideal leads. Its behavior as function of $\mathcal{E} / W^{4 / 3}$ is shown in Fig.3, where $\mathcal{E}$ is the Fermi energy measured from the lower band edge and $W$ is the amplitude of a random potential (all energies are measured in units of the hopping integral of the 1D Anderson model, see Eq.14 below). One can see that the parameter $\gamma$ is always finite but accepts small values in the deep of the allowed band, in accordance with the random phase approximation. Hence, finiteness of $\gamma$ is determined by the internal properties of the system and introduction of semi-transparent boundaries [12] is not actual.

In the plane $\left(\mathcal{E}, W^{2}\right)$ one can distinguish three characteristic domains (Fig.4): quasi-metallic $(|\gamma| \ll$ $1)$, strongly localized $(\gamma \gg 1)$ and "critical" $(\gamma \sim 1)$. If the energy $\mathcal{E}$ is varied for a fixed value of $W$, the distribution of conductance changes qualitatively and demonstrates something like the smoothed Anderson transition. The true phase transition arises in the limit $W \rightarrow 0$, where $\gamma \rightarrow 0$ and $\gamma \rightarrow \infty$ in the metallic and localized phase correspondingly, while the width of the critical region $(\gamma \sim 1)$ tends to zero.

Let now discuss the dependence of results on the properties of the ideal leads. We begin with a simple example, in order to demonstrate the existence of a subject for discussion. Removing the random 


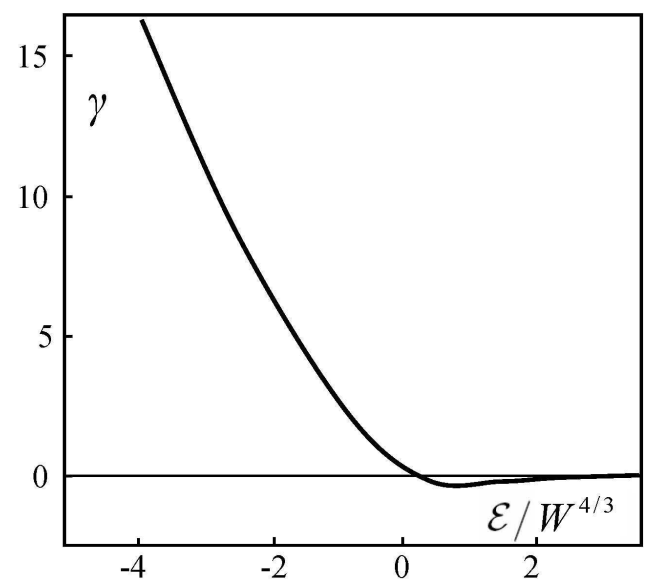

Figure 3: Parameter $\gamma$ in equation (2), corresponding to the limit of large $L$, as function of the energy $\mathcal{E}$, counted from the lower edge of the initial band.

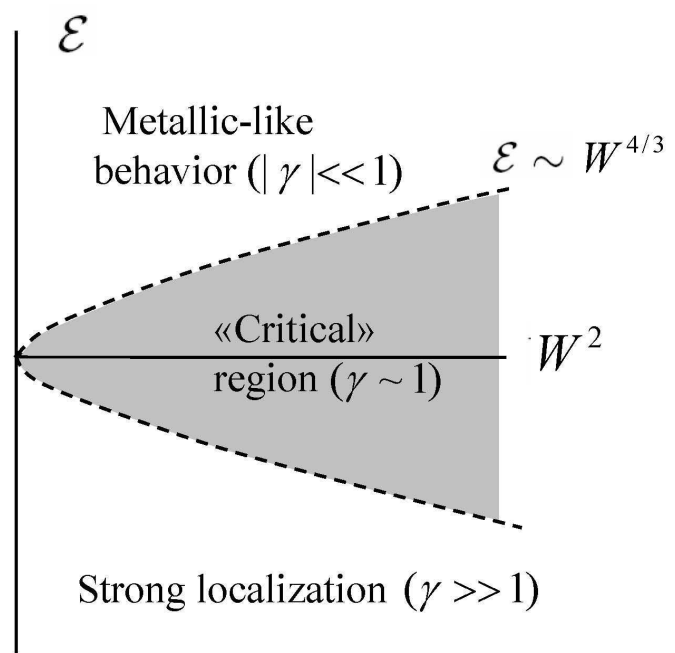

Figure 4: In the plane $\left(\mathcal{E}, W^{2}\right)$ one can distinguish three characteristic regions: quasi-metallic $(|\gamma| \ll 1)$, strongly localized $(\gamma \gg 1)$ and "critical" $(\gamma \sim 1)$. If the energy $\mathcal{E}$ is changed for a fixed amplitude $W$ of the random potential, then the conductance distribution undergoes qualitative changes resembling the smoothed Anderson transition. In the limit $W \rightarrow 0$ the true phase transition arises. potential in the situation of Fig.2,c, one has a simple quantum mechanical problem on the tunneling through the potential barrier 24]. Calculating the amplitudes of transmission $(t)$ and reflection $(r)$, one can find the Landauer resistance $\rho=|r|^{2} /|t|^{2}$ of the system

$$
\rho=\frac{1}{4}\left(\frac{k}{\kappa}+\frac{\kappa}{k}\right)^{2} \operatorname{sh}^{2} \kappa L,
$$

where $k$ is the Fermi momentum in the ideal leads, and $\kappa$ is the decrement of decay of the wave functions under the barrier. The $\rho$ dependence on $L$ is determined by the parameter $\kappa$, i.e. internal properties of the system, while the proportionality coefficient depends on $k$, i.e. the properties of the ideal leads.

This result contradicts to a physical intuition, so let us consider its interpretation. Our physical intuition was formed on the usual Ohmic regime, when the resistance $\rho$ is proportional to the system length $L$ or the number of scatterers $n$; hence, each scatterer gives the additive contribution to resistance. However, there exists the localization regime, where $\rho$ depends on $L$ exponentially, so $\ln \rho \propto L \propto n$ and each scatterer gives the multiplicative contribution to resistance. Since the ideal leads do not provide a dissipation, their contribution to resistance is related only with interfaces, and has a multiplicative character in the exponential regime. The latter changes the common coefficient in (4), which can vary from unity (for $k=\kappa$ ) till infinity.

Generally, the contribution of interfaces is not additive, nor multiplicative, and a situation is not trivial (Sec.8). In addition, the conductance of a finite system is a strongly fluctuating quantity [25, 26, and one should consider its distribution function. Dependence of the latter on the boundary conditions is manifested already in the metallic regime, where it can be investigated both analytically [27, 28], and numerically 29 .

Dependence of the conductance distribution of 1D systems on the properties of ideal leads is discussed in details in Sec.6. Let us declare several simple statements.

1. For large $L$ the distribution $P(\rho)$ is log-normal, and contains the limiting value of the parameter $\gamma$ at $L \rightarrow \infty$ :

$P(\rho)=\frac{1}{\rho \sqrt{4 \pi t}} \exp \left\{-\frac{[\ln \rho-(2 \gamma+1) t]^{2}}{4 t}\right\}, \quad t=\tilde{\alpha} L$. 
It is a solution of Eq. 2 for large $\rho$, when $1+2 \rho \approx 2 \rho$, $\rho(1+\rho) \approx \rho^{2}$. The parameters $\tilde{\alpha}$ and $\gamma$ can be established (Sec.5) using the exponents of growth for the second and fourth moments of the transfer matrix elements (Sec.4); they are determined by the internal properties of the system and do not depend on the ideal leads. The latter affect only the absolute scale of conductance.

2. In the deep of the forbidden band (Fig.2,c) the latter statement remains valid beyond the lognormal regime: the ideal leads do not affect the form of the distribution $P(\rho)$, but change the absolute scale of $\rho$,

$$
P(\rho) \rightarrow A P(A \rho), \quad A=1 / \Delta_{2}^{2},
$$

where the parameter $\Delta_{2}$ is defined below in Eq.9.

3. In the critical region, the situation is more complicated: the ideal leads change only the absolute scale of conductance, but the $A$ value in Eq. 6 is different in the log-normal regime and in the range of not very large $L$. In the latter case for the "natural" leads (Fig.2,a) one has the following distribution

$$
P(\rho)=\frac{1}{\Gamma(\gamma+1)} \frac{\rho^{\gamma} \exp \{-\rho / t\}}{t^{\gamma+1}},
$$

which is a solution of Eq.2 for small $\rho$, when $1+$ $2 \rho \approx 1, \rho(1+\rho) \approx \rho$. In the critical region one has $\gamma=-1 / 2$, while the parameter $A$ in Eq.6 takes a value $1 /\left(\Delta_{2}-\Delta_{1}\right)^{2}$, where $\Delta_{1}$ and $\Delta_{2}$ are defined in Eq.9.

4. The situation is even more complicated in the deep of the allowed band, where the ideal leads affect the whole distribution function in the range of not very large $L$. If for the "natural" leads (Fig.2,a) one has distribution (7) with $\gamma=0$, then for the foreign leads (Fig.2,b) its form is essentially modified: in particular, the universal distribution appears in the formal limit $L \rightarrow 0: 1$

$$
P(\rho)=\frac{1}{\pi} \sqrt{\frac{1}{\rho\left(\rho_{c}-\rho\right)}} \Theta\left(\rho_{c}-\rho\right),
$$

whose evolution with $L$ is demonstrated in Fig.5,a and Fig.5,b; the latter differ by the values of parameters

$$
\Delta_{1}=\frac{1}{2}\left(\frac{k}{\kappa}-\frac{\kappa}{k}\right), \quad \Delta_{2}=\frac{1}{2}\left(\frac{k}{\kappa}+\frac{\kappa}{k}\right) .
$$

\footnotetext{
${ }^{1}$ It should be stressed, that the limit $L \rightarrow 0$ is indeed formal, since the results (7) and (8) are restricted by the condition $L \gtrsim 1 / \kappa$.
}

Parameters (9) are defined for the forbidden band, but acquire the same form in the allowed band, if one set formally $\bar{k}=\kappa$ for the Fermi momentum $\bar{k}$ of the system under consideration; they are bounded by the relation

$$
\Delta_{2}^{2}-\Delta_{1}^{2}=1
$$

which is of vital significance for conservation of probability. Below we suggest for convenience that $\Delta_{1}$ is a free parameter, variating from $-\infty$ till $\infty$, while the positive parameter $\Delta_{2}$ is defined by Eq.10.

In $1 \mathrm{D}$ systems, the mean value of $\rho$ is usually not representative, but nevertheless is observable (Sec.8). Its evolution allows a complete description for arbitrary $L$ and gives a clear demonstration for the influence of the ideal leads. As function of $L$, both mean of $\rho$, and its higher moments can exhibit incommensurate oscillations, which provide the aperiodic character of oscillations for the resistance $\rho$ in the specific sample (Fig.8).

\section{Different types of transfer matrices}

The use of transfer matrices is the natural approach to investigation of $1 \mathrm{D}$ systems. The most convenient variant is the transfer matrix $T$ in the wave representation, which relates the amplitudes of waves on the left $\left(A e^{i k x}+B e^{-i k x}\right)$ and on the right $\left(C e^{i k x}+D e^{-i k x}\right)$ of a scatterer:

$$
\left(\begin{array}{l}
A \\
B
\end{array}\right)=T\left(\begin{array}{l}
C \\
D
\end{array}\right)
$$

It is determined by the transmission $(t)$ and reflection $(r)$ amplitudes and in the presence of the timereversal invariance allows the following parametrization 1

$T=\left(\begin{array}{cc}1 / t & -r / t \\ -r^{*} / t^{*} & 1 / t^{*}\end{array}\right)=\left(\begin{array}{cc}\sqrt{\rho+1} e^{i \varphi} & \sqrt{\rho} e^{i \theta} \\ \sqrt{\rho} e^{-i \theta} & \sqrt{\rho+1} e^{-i \varphi}\end{array}\right)$,

where $\rho=|r / t|^{2}$ is the Landauer resistance 22. For the successive arrangement of scatterers their transfer matrices are multiplicated. For a weak scatterer, the matrix $T$ is close to the unit one, which allows to derive the differential evolution equations for its elements and the Landauer resistance $\rho$.

For the energy in the forbidden band wave functions on the left $\left(A e^{\kappa x}+B e^{-\kappa x}\right)$ and on the right $\left(C e^{\kappa x}+D e^{-\kappa x}\right)$ of a scatterer are given by a superposition of the increasing and decreasing exponents 

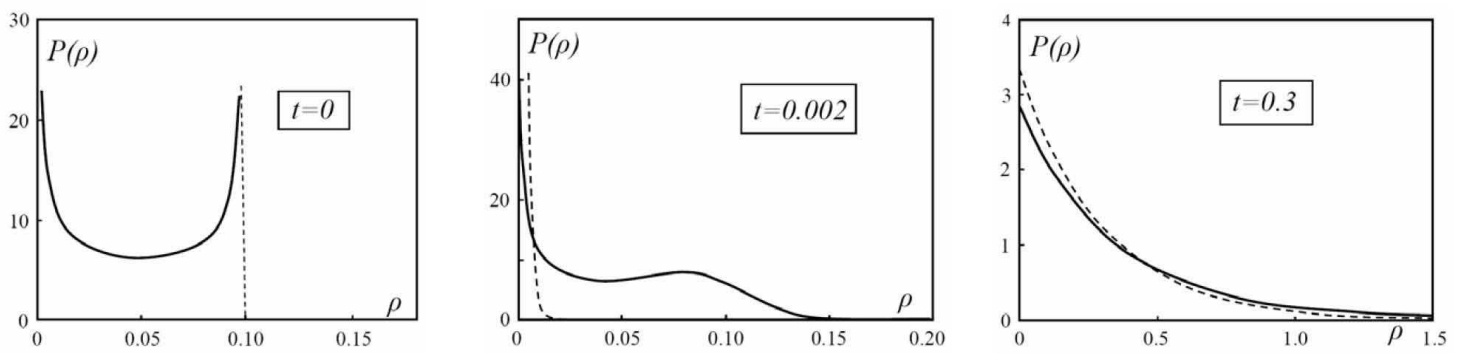

(a)
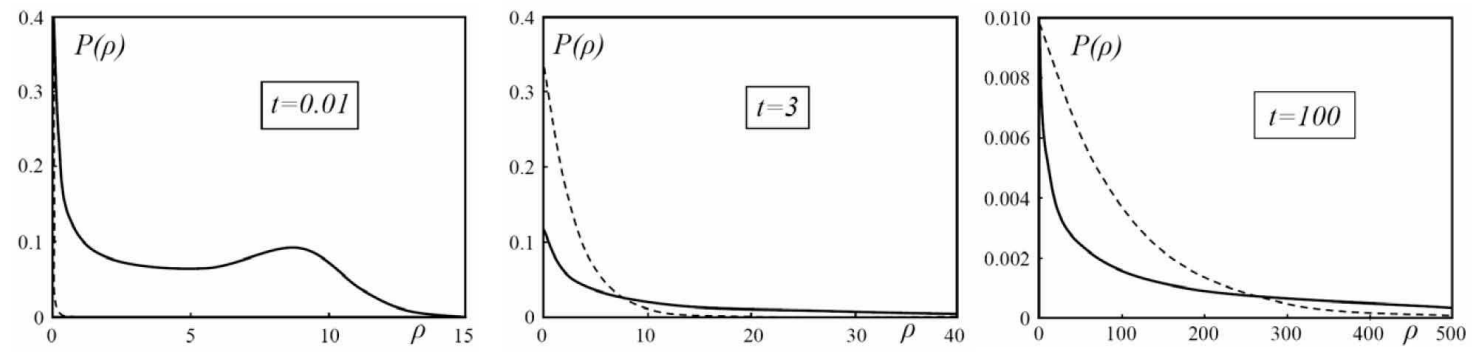

(b)

Figure 5: Evolution of the distribution $P(\rho)$ in the metallic regime for (a) the weak $\left(\Delta_{1}^{2}=0.1\right)$ and $(\mathrm{b})$ strong $\left(\Delta_{1}^{2}=10\right)$ difference of the Fermi levels in the given sample and the ideal leads. The dashed line shows the distribution $(7)$ with $\gamma=0$.

and can be related by the pseudo-transfer matrix

$$
\left(\begin{array}{l}
A \\
B
\end{array}\right)=\mathcal{T}\left(\begin{array}{l}
C \\
D
\end{array}\right)=\left(\begin{array}{ll}
t_{11} & t_{12} \\
t_{21} & t_{22}
\end{array}\right)\left(\begin{array}{l}
C \\
D
\end{array}\right),
$$

for which parametrization (12) is not valid and its relation with $\rho$ is not evident. The elements of the matrix $\mathcal{T}$ are real and its determinant is equal to unity, as in the case of (12).

At last, one can introduce the transfer matrix in the coordinate representation, as can be illustrated for the 1D Anderson model, describing by the discrete Schroedinger equation

$$
\Psi_{n+1}+\Psi_{n-1}+V_{n} \Psi_{n}=E \Psi_{n}
$$

where $E$ is the energy counted from the band center, and the hopping integral is set to be unity. Rewriting (14) in the form

$$
\left(\begin{array}{c}
\Psi_{n+1} \\
\Psi_{n}
\end{array}\right)=\left(\begin{array}{cc}
E-V_{n} & -1 \\
1 & 0
\end{array}\right)\left(\begin{array}{c}
\Psi_{n} \\
\Psi_{n-1}
\end{array}\right)
$$

and making $n$ iterations, one can obtain

$$
\left(\begin{array}{c}
\Psi_{n+1} \\
\Psi_{n}
\end{array}\right)=\tau\left(\begin{array}{c}
\Psi_{1} \\
\Psi_{0}
\end{array}\right)=\left(\begin{array}{ll}
\tau_{11} & \tau_{12} \\
\tau_{21} & \tau_{22}
\end{array}\right)\left(\begin{array}{c}
\Psi_{1} \\
\Psi_{0}
\end{array}\right)
$$

where the matrix $\tau$ is a product of $n$ matrices of type (15).

Three matrices $T, \mathcal{T}, \tau$ are determined by the internal properties of the system, and have their merits and drawbacks (Fig.6). These matrices do not allow to obtain the differential evolution equation for $\rho$, applicable for all energies. Indeed, the matrix $T$ possesses the necessary properties, but immediately applicable only in the allowed band; in the forbidden band its role is played by the pseudo-transfer matrix $\mathcal{T}$, which has no direct relation with $\rho$. The matrix $\tau$ is applicable for all energies, but has no direct relation with $\rho$ and is not close to the unit one for a weak scatterer. The matrices $\mathcal{T}$ and $\tau$ consist of the real elements, which has some technical advantages 2 .

To overcome these difficulties, let introduce the "external" matrix $\tilde{T}$, which takes into account the presence of the foreign leads (Fig.2,b) and is related with the "internal" matrix $T$ of the system with the help of the "edge" matrices $T_{a}$ and $T_{b}$ :

$\tilde{T}=T_{a} T T_{b}=\left(\begin{array}{cc}a & a_{1} \\ a_{1} & a\end{array}\right)\left(\begin{array}{ll}T_{11} & T_{12} \\ T_{21} & T_{22}\end{array}\right)\left(\begin{array}{ll}b & b_{1} \\ b_{1} & b\end{array}\right)$,

\footnotetext{
${ }^{2}$ In particular, the analysis of fourth moments for matrices with the complex elements looks rather hopeless, since it demands diagonalization of the matrix of large size.
} 
Matrix $T$

\begin{tabular}{||c|c||}
\hline Drawbacks & Merits \\
\hline $\begin{array}{c}\text { Restricted } \\
\text { by the allowed } \\
\text { band }\end{array}$ & $\begin{array}{c}\text { Determinate by internal } \\
\text { properties of the system }\end{array}$ \\
& $\begin{array}{c}\text { Direct relation with } \rho \\
\text { Close to the unit matrix } \\
\text { for a weak scatterer }\end{array}$ \\
\hline
\end{tabular}

Matrix $\tau$

\begin{tabular}{|c|c|}
\hline Drawbacks & Merits \\
\hline $\begin{array}{l}\text { Not close to } \\
\text { the unit matrix } \\
\text { for a weak } \\
\text { scatterer }\end{array}$ & $\begin{array}{c}\text { Determinate by internal } \\
\text { properties of the system } \\
\text { Applicable for all } \\
\text { energies }\end{array}$ \\
\hline $\begin{array}{l}\text { No direct } \\
\text { relation with } \rho\end{array}$ & Real matrix elements \\
\hline
\end{tabular}

Matrix $\mathcal{T}$

\begin{tabular}{||c|c||}
\hline Drawbacks & Merits \\
\hline $\begin{array}{c}\text { Restricted by } \\
\text { the forbidden } \\
\text { band }\end{array}$ & $\begin{array}{c}\text { Determinate by internal } \\
\text { properties of the system }\end{array}$ \\
$\begin{array}{c}\text { No direct } \\
\text { for a to the unit matrix } \\
\text { relation with } \rho\end{array}$ & Real matrix elements \\
\hline
\end{tabular}

Matrix $\tilde{T}$

\begin{tabular}{||c|c||}
\hline Drawbacks & Merits \\
\hline $\begin{array}{c}\text { Depends on } \\
\text { the ideal leads } \\
\text { energies }\end{array}$ & $\begin{array}{c}\text { Direct relation with } \rho \\
\text { Close to the unit matrix } \\
\text { for a weak scatterer }\end{array}$ \\
\hline
\end{tabular}

Figure 6: Merits and drawbacks of different transfer matrices.

$$
\begin{aligned}
a=\frac{1}{2}\left(1+\frac{\bar{k}}{k}\right), \quad a_{1}=\frac{1}{2}\left(1-\frac{\bar{k}}{k}\right), \\
b=\frac{1}{2}\left(1+\frac{k}{\bar{k}}\right), \quad b_{1}=\frac{1}{2}\left(1-\frac{k}{\bar{k}}\right) .
\end{aligned}
$$

The edge matrices have a simple sense: for example, $T_{a}$ relates the amplitudes of waves on the left of the interface $\left(A e^{i k x}+B e^{-i k x}\right)$ and on the right of it $\left(A_{1} e^{i \bar{k} x}+B_{1} e^{-i \bar{k} x}\right)$. One can see that $T_{a} T_{b}=1$, i.e. the edge matrices are are mutually inverse.

For the energy in the forbidden band (Fig.2,c) the explicit introduction of ideal leads is inevitable, and is also realized with the help of the edge matrices, transforming the pseudo-transfer matrix $\mathcal{T}$ to the true transfer matrix $\tilde{T}$

$$
\begin{gathered}
\tilde{T}=T_{a} \mathcal{T} T_{b}=\left(\begin{array}{cc}
a & a^{*} \\
a^{*} & a
\end{array}\right)\left(\begin{array}{cc}
t_{11} & t_{12} \\
t_{21} & t_{22}
\end{array}\right)\left(\begin{array}{cc}
b & b^{*} \\
b^{*} & b
\end{array}\right), \\
a=\frac{1}{2}\left(1+\frac{\kappa}{i k}\right), \quad b=\frac{1}{2}\left(1+\frac{i k}{\kappa}\right) .
\end{gathered}
$$

Relations (18) can be obtained from (17) by the change $\bar{k} \rightarrow-i \kappa$.

The use of the edge matrices allows also to relate $\tilde{T}$ and $\tau$

$$
\tilde{T}=\frac{1}{2 i \sin k a_{0}}\left(\begin{array}{cc}
1 & -\mathrm{e}^{-i k a_{0}} \\
-1 & \mathrm{e}^{i k a_{0}}
\end{array}\right) .
$$

$$
\cdot\left(\begin{array}{ll}
\tau_{11} & \tau_{12} \\
\tau_{21} & \tau_{22}
\end{array}\right)\left(\begin{array}{cc}
\mathrm{e}^{i k a_{0}} & \mathrm{e}^{-i k a_{0}} \\
1 & 1
\end{array}\right)
$$

where $a_{0}$ is the lattice constant for the model (14). One can see that the explicit introduction of the ideal leads corresponds to the linear transformation of matrix elements. A linear transformation does not change the exponents of growth for the second and fourth moments, which are determined by the internal properties of the system and do not depend on the ideal leads (Sec.4). As should be clear from Fig.6, the matrix $\tilde{T}$ possesses all necessary properties, but depends on the ideal leads; the latter can be consider as a drawback, but in fact it reflects the objective reality.

\section{Succession of point scatterers}

The coefficients $\tilde{\alpha}, \gamma_{1}, \gamma_{2}$ of equation (3) are not necessary constant and can variate in the course of evolution. To gain an insight into the character of this evolution, let consider a succession of point scatterers. For definiteness, let have in mind the Anderson model (14) near the band edge, where it corresponds to discretization of the usual Schroedinger equation. 
One can easily verify that the point scatterer with the potential $V \delta_{n 0}$ is described by the transfer matrix

$$
\left(\begin{array}{cc}
1-i \epsilon & -i \epsilon \\
i \epsilon & 1+i \epsilon
\end{array}\right), \quad \text { where } \quad \epsilon=\frac{V}{2 k a_{0}} .
$$

If two scatterers with amplitudes $V_{0}$ and $V_{1}$ are arranged at the distance $L$, then the transfer matrix arises

$$
\begin{array}{r}
\left(\begin{array}{cc}
1-i \epsilon_{0} & -i \epsilon_{0} \\
i \epsilon_{0} & 1+i \epsilon_{0}
\end{array}\right)\left(\begin{array}{cc}
\mathrm{e}^{-i k L} & 0 \\
0 & \mathrm{e}^{i k L}
\end{array}\right) \\
\cdot\left(\begin{array}{cc}
1-i \epsilon_{1} & -i \epsilon_{1} \\
i \epsilon_{1} & 1+i \epsilon_{1}
\end{array}\right) .
\end{array}
$$

At last, if the scatterers with amplitudes $V_{0}, V_{1}, V_{2}, \ldots, V_{n}$ are arranged at the points 0 , $L_{1}, L_{1}+L_{2}, \ldots$, then the corresponding transfer matrix has a form

$$
T^{(n)}=T_{\epsilon_{0}} T_{\delta_{1}} T_{\epsilon_{1}} T_{\delta_{2}} T_{\epsilon_{2}} \ldots T_{\delta_{n}} T_{\epsilon_{n}}
$$

where

$$
\begin{aligned}
& T_{\epsilon_{s}}=\left(\begin{array}{cc}
1-i \epsilon_{s} & -i \epsilon_{s} \\
i \epsilon_{s} & 1+i \epsilon_{s}
\end{array}\right), \quad \epsilon_{s}=\frac{V_{s}}{2 k a_{0}}, \\
& T_{\delta_{s}}=\left(\begin{array}{cc}
\mathrm{e}^{-i \delta_{s}} & 0 \\
0 & \mathrm{e}^{i \delta_{s}}
\end{array}\right), \quad \delta_{s}=k L_{s} .
\end{aligned}
$$

If the number of scatterers is increased by unity, one has the recurrent relation

$$
\begin{gathered}
T^{(n)}=T^{(n-1)}\left(\begin{array}{cc}
u_{n} & v_{n} \\
v_{n}^{*} & u_{n}^{*}
\end{array}\right), \\
u_{n}=\left(1-i \epsilon_{n}\right) \mathrm{e}^{-i \delta_{n}}, \quad v_{n}=-i \epsilon_{n} \mathrm{e}^{-i \delta_{n}} .
\end{gathered}
$$

For convenience we set $\epsilon_{0}=0$, in order to use the unit matrix as the initial condition for $T^{(0)}$. If all $\epsilon_{n}$ are small, then for not very large $n$ one can retain two first orders in $v_{n}$; producing direct multiplication of matrices, one has

$$
\begin{gathered}
T_{11}^{(n)}=u_{1} u_{2} \ldots u_{n}+\sum_{i=1}^{n-1} \sum_{j=i+1}^{n} u_{1} u_{2} \ldots u_{i-1} v_{i} \\
\cdot u_{i+1}^{*} \ldots u_{j-1}^{*} v_{j}^{*} u_{j+1} \ldots u_{n} \\
T_{12}^{(n)}=\sum_{i=1}^{n} u_{1} u_{2} \ldots u_{i-1} v_{i} u_{i+1}^{*} \ldots u_{n}^{*}
\end{gathered}
$$

and $T_{21}=T_{12}^{*}, T_{22}=T_{11}^{*}$.
In the Anderson model (14), a scatterer is present at each site of the lattice, so all $\delta_{s}$ are equal, $\delta_{s}=$ $k a_{0}$. Expressions (25) can be reduced to the form

$T_{11}^{(n)}=\sqrt{1+S_{1}^{2}+S_{2}^{2}} \mathrm{e}^{-i S-i n \delta}, \quad T_{12}^{(n)}=S_{1}-i S_{2}$,

where in the main order in $\epsilon$

$$
\begin{gathered}
S=\sum_{s=1}^{n} \epsilon_{s}, \quad S_{1}=\sum_{s=1}^{n} \epsilon_{s} \sin (n-2 s) \delta, \\
S_{2}=\sum_{s=1}^{n} \epsilon_{s} \cos (n-2 s) \delta .
\end{gathered}
$$

Accepting as usually for the Anderson model

$$
\left\langle V_{n}\right\rangle=0, \quad\left\langle V_{n}^{2}\right\rangle=W^{2}
$$

one has the zero means for all sums in (27), while for the second moments

$$
\begin{gathered}
\left\langle S^{2}\right\rangle=2\left\langle S_{1}^{2}\right\rangle=2\left\langle S_{2}^{2}\right\rangle=\epsilon^{2} n, \\
\left\langle S S_{1}\right\rangle \sim\left\langle S S_{2}\right\rangle \sim\left\langle S_{1} S_{2}\right\rangle \sim \epsilon^{2},
\end{gathered}
$$

where $\epsilon^{2}=W^{2} / 4 k^{2} a_{0}^{2}$. Above expressions are valid under condition

$$
1 / \delta \ll n \ll 1 / \epsilon^{2},
$$

where the left inequality provides the large number of oscillations of sine and cosine in sums (27), while the right inequality is necessary for neglection of higher orders in $\epsilon$. For large $n$, all sums (27) are normally distributed and practically uncorrelated, so their mutual distribution function has a form

$$
P\left(S, S_{1}, S_{2}\right) \sim \exp \left\{-\frac{S^{2}}{2 n \epsilon^{2}}-\frac{S_{1}^{2}}{n \epsilon^{2}}-\frac{S_{2}^{2}}{n \epsilon^{2}}\right\} .
$$

Using the relations $\rho=S_{1}^{2}+S_{2}^{2}, \varphi=-S-n \delta$, one has the mutual distribution for the elements of the transfer matrix (12)

$$
P(\rho, \varphi, \theta) \sim \exp \left\{-\frac{(\varphi+n \delta)^{2}}{2 n \epsilon^{2}}-\frac{\rho}{n \epsilon^{2}}\right\} .
$$

It should be clear, that for $\delta \gg \epsilon^{2}$, i.e. in the deep of the allowed band, the phase $\theta$ becomes completely random on the scale $n \sim 1 / \delta$. Stochastization of the phase $\varphi$ occurs on the scale $n \sim 1 / \epsilon^{2}$, but its uniform distribution arises already on the scale $1 / \delta$ due to regular variations. In fact, stochastization of $\theta$ is sufficient for applicability of the random phase 
approximation and disappearance of $\gamma$, since the evolution equation for $P(\rho)$ contains only the combination $\psi=\theta-\varphi$ (see Sec.7). Solution of Eq.2 for small $\rho$ has a form (7), which agrees with (32) for $\gamma=0$ and $t=\epsilon^{2} n$; the latter relations follow from Eq.93 of Sec.7. For large $n$ the exponential growth of the elements $T_{i j}$ is determined by the product $\left|u_{1}\right|\left|u_{2}\right| \ldots\left|u_{n}\right| \equiv \mathrm{e}^{w_{n}}$, which is contained in all terms of (25), as well as in the higher order terms. The quantity $w_{n}$ has the mean value $\epsilon^{2} n / 2$, which (in view $\rho=\left|T_{12}\right|^{2}$ ) agrees with the result $a=\epsilon^{2}$ for the parameter $a$ in the log-normal distribution (Sec. 5 ). Neglected terms do not give contributions $O\left(\epsilon^{2}\right)$ in the mean of $w_{n}$, but change its variance.

Above considerations are valid in the deep of the allowed band for the "natural" ideal leads (Fig.2,a). The forbidden band is described by the transfer matrix $\tilde{T}^{(n)}=T_{a} \mathcal{T}^{(n)} T_{b}$, where the pseudo-transfer matrix $\mathcal{T}^{(n)}$ is determined by the above relations with the change $k \rightarrow-i \kappa$; in particular

$$
\begin{gathered}
\mathcal{T}^{(n)}=\mathcal{T}^{(n-1)}\left(\begin{array}{cc}
u_{n} & v_{n} \\
v_{n}^{*} & u_{n}^{*}
\end{array}\right), \\
u_{n}=\left(1+\epsilon_{n}\right) \mathrm{e}^{-\delta_{n}}, \quad v_{n}=\epsilon_{n} \mathrm{e}^{-\delta_{n}}, \\
\epsilon_{n}=V_{n} / 2 \kappa a_{0}, \quad \delta_{n}=\kappa L_{n},
\end{gathered}
$$

where the star indicates not the complex conjugation, but a change of signs for $\epsilon_{n}$ and $\delta_{n}$. With this modification, relations (25) remain formally valid; extracting the factor $u_{1}^{*} u_{2}^{*} \ldots u_{n}^{*} \equiv \exp \left(w_{n}\right)$ from all sums, one obtains

$$
\begin{aligned}
& \mathcal{T}^{(n)}=\left(\begin{array}{cc}
\mathrm{e}^{-\tilde{w}_{n}}-\mathrm{e}^{\tilde{w}_{n}} S_{1} S_{2} & \mathrm{e}^{\tilde{w}_{n}} S_{1} \\
-\mathrm{e}^{\tilde{w}_{n}} S_{2} & \mathrm{e}^{\tilde{w}_{n}}
\end{array}\right), \\
& S_{1}=\sum_{s=1}^{n} \epsilon_{s} \mathrm{e}^{-2 s \delta}, \quad S_{2}=\sum_{s=0}^{n-1} \epsilon_{n-s} \mathrm{e}^{-2 s \delta},
\end{aligned}
$$

where $\tilde{w}_{n}$ differs from $w_{n}$ by the contribution $O\left(\epsilon^{2}\right)$ with a zero mean, while $S_{1}$ and $S_{2}$ are given in the main order in $\epsilon$. Substitution to (18) gives for the phase variables

$$
\begin{array}{r}
\operatorname{tg} \varphi=-\Delta_{1}-\Delta_{2}\left(S_{1}+S_{2}\right), \\
\operatorname{ctg} \theta=\left(S_{1}-S_{2}\right) / \Delta_{2},
\end{array}
$$

where $\Delta_{1}$ and $\Delta_{2}$ are defined in (9). Having in mind that under condition (30)

$$
\left\langle S_{1}^{2}\right\rangle=\left\langle S_{2}^{2}\right\rangle=\epsilon^{2} / 4 \delta, \quad\left\langle S_{1} S_{2}\right\rangle \approx 0
$$

we see that fluctuations of $\varphi$ and $\theta$ are restricted and do not increase with $n$. The case $k=\kappa$ is special, since $\Delta_{1}=0$ and $\Delta_{2}=1$, so

$$
\varphi=-\left(S_{1}+S_{2}\right), \quad \theta=\pi / 2+\left(S_{2}-S_{1}\right),
$$

and the variable $\psi=\theta-\varphi$ does not have an essential evolution, being localized near $\pi / 2$ for all $n \lesssim 1 / \epsilon^{2}$. As shown in Sec.7, this property remains valid for $n \gtrsim 1 / \epsilon^{2}$. For large $n$ the exponent $\mathrm{e}^{\tilde{w}_{n}}$ provides the growth of elements $t_{i j}$ and correspondingly $\rho \sim \mathrm{e}^{2 \tilde{w}_{n}}$; the quantity $2 \tilde{w}_{n}$ has the mean $\left(2 \delta-\epsilon^{2}\right) n$ and the variance $4 \epsilon^{2} n$, in agreement with Eq.56 of Sec.5.

Let come to the critical region, determined by the condition $\delta \ll \epsilon^{2}$. Then for sufficiently small $n$ one can set $\delta_{s}=0$ in Eq.23 and obtain

$$
T^{(n)}=\left(\begin{array}{cc}
1-i S & -i S \\
i S & 1+i S
\end{array}\right), \quad S=\sum_{s=1}^{n} \epsilon_{s},
$$

where $S$ has the Gaussian distribution analogously to (31). The mutual distribution for $\rho, \varphi, \theta$ is given by the relation

$$
\begin{gathered}
P(\rho, \varphi, \theta)=\int d S \frac{1}{\sqrt{2 \pi n \epsilon^{2}}} \exp \left(-\frac{S^{2}}{2 n \epsilon^{2}}\right) . \\
\cdot \delta\left(\rho-S^{2}\right) \delta(\varphi+\operatorname{arctg} S) \delta\left(\theta+\frac{\pi}{2}\right)
\end{gathered}
$$

and integration over $\varphi$ and $\theta$ gives the result

$$
P(\rho)=\frac{1}{\sqrt{\rho}} \frac{1}{\sqrt{2 \pi n \epsilon^{2}}} \exp \left(-\frac{\rho}{2 n \epsilon^{2}}\right),
$$

which agrees with $(7)$ for $\gamma=-1 / 2, t=2 n \epsilon^{2}$; the latter follows from Eq.93 of Sec.7, if $\psi$ is localized near $\pm \pi / 2$. Such localization is indeed valid for small $\rho$, when $\varphi \sim \sqrt{\rho} \ll 1, \theta=-\pi / 2$, and $(40)$ is a solution of Eq.2. However, the result (40) remains valid in the more wide interval $n \lesssim\left(\epsilon^{2} \delta^{2}\right)^{-1 / 3}$ (Sec.8), when $\rho \sim n \epsilon^{2}$ can be large; in this case $\varphi$ is localized near $\pm \pi / 2$, while $\sin \psi$ becomes small in agreement with the results for the log-normal regime (Secs.5, 7).

\section{Evolution of moments}

According to (33), the elements of the pseudotransfer matrix in the forbidden band obey the evolution equations

$$
t_{11}^{(n)}=u_{n} t_{11}^{(n-1)}+v_{n}^{*} t_{12}^{(n-1)}, \quad t_{12}^{(n)}=v_{n} t_{11}^{(n-1)}+u_{n}^{*} t_{12}^{(n-1)},
$$


and the analogous equations for $t_{21}^{(n)}$ and $t_{22}^{(n)}$; it is essential that $t_{i j}^{(n-1)}$ do not contain $\epsilon_{n}$ and are statistically independent of $u_{n}, v_{n}$. Introducing the notations for the second moments

$$
z_{1}^{(n)}=\left\langle\left[t_{11}^{(n)}\right]^{2}\right\rangle, \quad z_{2}^{(n)}=\left\langle t_{11}^{(n)} t_{12}^{(n)}\right\rangle, \quad z_{3}^{(n)}=\left\langle\left[t_{12}^{(n)}\right]^{2}\right\rangle,
$$

one can obtain the system of the linear difference equations with constant coefficients

$$
\left(\begin{array}{c}
z_{1}^{(n)} \\
z_{2}^{(n)} \\
z_{3}^{(n)}
\end{array}\right)=\left(\begin{array}{ccc}
1-2 \delta+\epsilon^{2} & -2 \epsilon^{2} & \epsilon^{2} \\
\epsilon^{2} & 1-2 \epsilon^{2} & \epsilon^{2} \\
\epsilon^{2} & -2 \epsilon^{2} & 1+2 \delta+\epsilon^{2}
\end{array}\right)\left(\begin{array}{c}
z_{1}^{(n-1)} \\
z_{2}^{(n-1)} \\
z_{3}^{(n-1)}
\end{array}\right)
$$

whose solution is sought in the exponential form, $z_{i}^{(n)} \sim \lambda^{n}[\underline{30}$; it is easy to see that $\lambda$ is an eigenvalue of the matrix (43). Setting $\lambda=1+x$, one has the equation for $x$

$$
x\left(x^{2}-4 \delta^{2}\right)=8 \epsilon^{2} \delta^{2} .
$$

We have in mind the limiting transition

$$
\delta \rightarrow 0, \quad \epsilon \rightarrow 0, \quad \delta / \epsilon^{2}=\text { const },
$$

which is actual near the band edge of the ideal crystal, where one can neglect the effects of commensurability [32, 33 complicating the analysis; correspondingly, we retain the terms of the first order in $\delta$ and the second order in $\epsilon$ in the matrix (43).

Analogously we set for the fourth moments

$$
\begin{gathered}
z_{1}^{(n)}=\left\langle\left[t_{11}^{(n)}\right]^{4}\right\rangle, \quad z_{2}^{(n)}=\left\langle\left[t_{11}^{(n)}\right]^{3} t_{12}^{(n)}\right\rangle, \quad z_{3}^{(n)}=\left\langle\left[t_{11}^{(n)}\right]^{2}\left[t_{12}^{(n)}\right]^{2}\right\rangle, \\
z_{4}^{(n)}=\left\langle t_{11}^{(n)}\left[t_{12}^{(n)}\right]^{3}\right\rangle, \quad z_{5}^{(n)}=\left\langle\left[t_{12}^{(n)}\right]^{4}\right\rangle,
\end{gathered}
$$

and obtain the system of difference equations

$$
\left(\begin{array}{c}
z_{1}^{(n)} \\
z_{2}^{(n)} \\
z_{3}^{(n)} \\
z_{4}^{(n)} \\
z_{5}^{(n)}
\end{array}\right)=\left(\begin{array}{ccccc}
1-4 \delta+6 \epsilon^{2} & -12 \epsilon^{2} & 6 \epsilon^{2} & 0 & 0 \\
3 \epsilon^{2} & 1-2 \delta-3 \epsilon^{2} & -3 \epsilon^{2} & 3 \epsilon^{2} & 0 \\
\epsilon^{2} & 2 \epsilon^{2} & 1-6 \epsilon^{2} & 2 \epsilon^{2} & \epsilon^{2} \\
0 & 3 \epsilon^{2} & -3 \epsilon^{2} & 1+2 \delta-3 \epsilon^{2} & 3 \epsilon^{2} \\
0 & 0 & 6 \epsilon^{2} & -12 \epsilon^{2} & 1+4 \delta+6 \epsilon^{2}
\end{array}\right)\left(\begin{array}{c}
z_{1}^{(n-1)} \\
z_{2}^{(n-1)} \\
z_{3}^{(n-1)} \\
z_{4}^{(n-1)} \\
z_{5}^{(n-1)}
\end{array}\right)
$$

Accepting $z_{i}^{(n)} \sim \lambda^{n}$ and setting $\lambda=1+x$, we have the equation for $x$

$$
x\left(x^{2}-4 \delta^{2}\right)\left(x^{2}-16 \delta^{2}\right)=24 \epsilon^{2}\left(7 \delta^{2} x^{2}-16 \delta^{4}\right) .
$$

Curiously, equations (44) and (48) can be obtained, if one composes the product of diagonal elements of matrices (43), (47) and retains the terms of the second order in $\epsilon$. Indeed, non-diagonal elements give contributions $O\left(\epsilon^{4}\right)$ and higher, whose concellation can be foreseen beforehand. Since $\epsilon^{2}=W^{2} / 4 \delta^{2}$ (see Sec.3), the combinations $\epsilon^{2}, \epsilon^{4}, \ldots, \epsilon^{10}$ and $\delta^{2} \epsilon^{4}, \delta^{2} \epsilon^{6}$ contain singularities at $\delta \rightarrow 0$, whose absence is evident from the evolution equations for the coordinate transfer matrix (see Appendix 1); only combinations $\delta^{2} \epsilon^{2}$ and $\delta^{4} \epsilon^{2}$ are allowable, which enters in Eqs.44,48. 3 In the deep of the allowed or forbidden band, the possibility of restriction by diagonal elements allows to establish the exponents of growth for higher moments and verify their accordance to the log-normal distribution.

\footnotetext{
${ }^{3}$ We have listed all possible combinations. Indeed, a change of the $\delta$ sign in (43), (47) leads to the analogous matrices, which can be transformed to the initial form, if the components of columns are renumerated in the inverse order; so the odd powers of $\delta$ do not appear. Since we consider the limit $\delta \sim \epsilon^{2} \rightarrow 0$ (see Eq.45), then only combinations $\delta^{2 n} \epsilon^{2 m}$ with $4 n+2 m \leq 6$ for (43) and $4 n+2 m \leq 10$ for (47) are possible; among them only combinations with $n \geq m$ do not have singularities for $\delta \rightarrow 0$.
} 


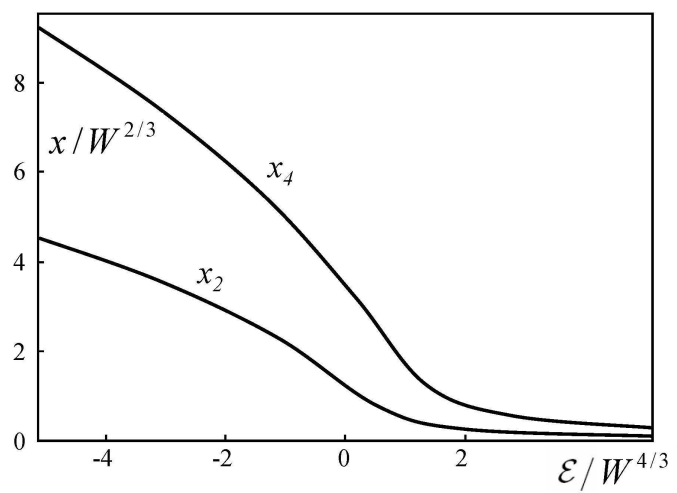

Figure 7: The positive root of the first $\left(x_{2}\right)$ and second $\left(x_{4}\right)$ equation $(49)$ against the energy $\mathcal{E}$.

For the Anderson model (14) one can set $\delta^{2}=-\mathcal{E}$, $4 \epsilon^{2} \delta^{2}=W^{2}$, where $\mathcal{E}$ is the energy counted from the lower band edge; correspondingly, equations (44), (48) can be rewritten in the form

$$
\begin{gathered}
x\left(x^{2}+4 \mathcal{E}\right)=2 W^{2} \\
x\left(x^{2}+4 \mathcal{E}\right)\left(x^{2}+16 \mathcal{E}\right)=42 W^{2} x^{2}+96 W^{2} \mathcal{E} .
\end{gathered}
$$

Equations (49) were derived for $\mathcal{E}<0$, but can be analytically continued to arbitrary $\mathcal{E}$ due to regularity in $\mathcal{E}$. These equations can be derived also using the coordinate transfer matrix (see Appendix 1), which is applicable for arbitrary $\mathcal{E}$ and does not require the analytical continuation. One of the roots for each of equations (49) remains positive for all physical values of parameters; it has the maximal real part between all roots of the equation and determines the exponent of growth for the second $\left(x_{2}\right)$ or fourth $\left(x_{4}\right)$ moments. Behavior of $x_{2}$ and $x_{4}$ against $\mathcal{E} / W^{4 / 3}$ is shown in Fig.7.

It is not difficult to obtain the asymptotic results for $x_{2}$ and $x_{4}$. In the deep of the forbidden band $\left(\delta \gg \epsilon^{2}\right)$ we have from $(44),(48)$

$$
x_{2}=2 \delta+\epsilon^{2}, \quad x_{4}=4 \delta+6 \epsilon^{2} .
$$

To come into the allowed band, one makes the replacements $\delta \rightarrow i \delta, \epsilon \rightarrow-i \epsilon$ in Eqs.44,48, and in the deep of the band $\left(\delta \gg \epsilon^{2}\right)$ finds the results

$$
x_{2}=2 \epsilon^{2}, \quad x_{4}=6 \epsilon^{2} .
$$

In the critical region $\left(\delta \ll \epsilon^{2}\right)$ one has

$$
x_{2}=2\left(\epsilon^{2} \delta^{2}\right)^{1 / 3}, \quad x_{4}=2\left(21 \epsilon^{2} \delta^{2}\right)^{1 / 3} .
$$

In terms of $\mathcal{E}$ and $W$ we can write

$$
\begin{gathered}
x_{2}=2|\mathcal{E}|^{1 / 2}+W^{2} / 4|\mathcal{E}|, \\
x_{4}=4|\mathcal{E}|^{1 / 2}+3 W^{2} / 2|\mathcal{E}| \text { for }-\mathcal{E} \gg W^{4 / 3}, \\
x_{2}=\left(2 W^{2}\right)^{1 / 3}, x_{4}=\left(42 W^{2}\right)^{1 / 3} \text { for }|\mathcal{E}| \ll W^{4 / 3}, \\
x_{2}=W^{2} / 2 \mathcal{E}-W^{6} / 32 \mathcal{E}^{4}, \\
x_{4}=3 W^{2} / 2 \mathcal{E}+27 W^{6} / 64 \mathcal{E}^{4} \text { for } \mathcal{E} \gg W^{4 / 3},
\end{gathered}
$$

where the latter result is given with higher accuracy.

\section{Parameters of the log-normal distribution}

For large $L=n a_{0}$ the distribution $P(\rho)$ is lognormal

$$
P(\rho)=\frac{1}{\rho \sqrt{2 \pi b n}} \exp \left\{-\frac{(\ln \rho-a n)^{2}}{2 b n}\right\},
$$

as was established in many papers (1], 13]-18, [21]-24]) for partial cases, and follows most generally from the modified Dorokhov-Mello-PereyraKumar equation 31]; in the presence of foreign leads it is derived in Sec.7. The moments of the distribution (52) can be easily found, $\left\langle\rho^{m}\right\rangle=\mathrm{e}^{a m n+b m^{2} n / 2}$. Since $\rho$ is determined by the expression, quadratic in the transfer matrix elements, the parameters $a$ and $b$ can be established by comparison with the growth of the second and fourth moments

$$
\begin{gathered}
\langle\rho\rangle=\mathrm{e}^{a n+\frac{1}{2} b n}=C_{1} \mathrm{e}^{x_{2} n}, \\
\left\langle\rho^{2}\right\rangle=\mathrm{e}^{2 a n+2 b n}=C_{2} \mathrm{e}^{x_{4} n} .
\end{gathered}
$$

It is easy to see that

$$
a=2 x_{2}-\frac{1}{2} x_{4}, \quad b=x_{4}-2 x_{2},
$$

while for the parameter $\gamma$ in (5)

$$
\gamma=\frac{a}{b}-\frac{1}{2}=\frac{3 x_{2}-x_{4}}{x_{4}-2 x_{2}} .
$$

Substitution of the asymptotic expressions (50) gives

$$
\begin{aligned}
& a=2 \delta-\epsilon^{2}, \quad b=4 \epsilon^{2} \quad \text { (forbidden band) } \\
& a=\epsilon^{2}, \quad b=2 \epsilon^{2} \quad \text { (allowed band) }
\end{aligned}
$$

$a=1.24\left(\epsilon^{2} \delta^{2}\right)^{1 / 3}, \quad b=1.52\left(\epsilon^{2} \delta^{2}\right)^{1 / 3} \quad$ (critical region) 
The use of asymptotic forms (51) gives for the parameter $\gamma$ in (5)

$$
\gamma=\left\{\begin{array}{cc}
2|\mathcal{E}|^{3 / 2} / W^{2}, & -\mathcal{E} \gg W^{4 / 3} \\
0.316, & |\mathcal{E}| \ll W^{4 / 3} \\
-33 W^{4} / 32 \mathcal{E}^{3}, & \mathcal{E} \gg W^{4 / 3}
\end{array},\right.
$$

while the parameter $\tilde{\alpha}$ is related with $b$ according to $\tilde{\alpha}=b / 2 a_{0}$.

As shown in Sec.2, introduction of the foreign leads results in the linear transformation for the elements of the transfer matrix, which does not change the exponents $x_{2}$ and $x_{4}$ for the second and fourth moments. By this reason, the latter do not depend on the properties of the ideal leads, as well as parameters $a, b, \gamma$. The ideal leads affect only the coefficients $C_{1}$ and $C_{2}$ in Eq.53, which change the absolute scale of $\rho$ and the origin of $n$.

\section{Dependence of $P(\rho)$ on the ideal leads}

According to (12) the elements of the transfer matrix $T$ obey the relations $T_{22}=T_{11}^{*}, T_{21}=T_{12}^{*}$, following from the time reversal invariance; so it is sufficient to consider the elements

$$
T_{11}=x+i y, \quad T_{12}=z+i \omega .
$$

If the mutual distribution $P(\rho, \varphi, \theta)$ is known for the parameters of the matrix (12), then the distribution of $x, y, z, \omega$ is composed according to the rule

$$
\begin{aligned}
& P_{1}(x, y, z, \omega)=\int d \rho d \varphi d \theta P(\rho, \varphi, \theta) . \\
& \cdot \delta(x-\sqrt{1+\rho} \cos \varphi) \delta(y-\sqrt{1+\rho} \sin \varphi) \\
& \cdot \delta(z-\sqrt{\rho} \cos \theta) \delta(\omega-\sqrt{\rho} \sin \theta) .
\end{aligned}
$$

Setting $\rho=r^{2}, 1+\rho=R^{2}$ and introducing decomposition of unity under integration

$$
1=\int d R^{2} \delta\left(R^{2}-r^{2}-1\right),
$$

it is easy to find the relation between $P_{1}(x, y, z, \omega)$ and $P(\rho, \varphi, \theta)$ :

$$
\begin{aligned}
P_{1}(x, y, z, \omega)= & P\left(z^{2}+\omega^{2}, \operatorname{Arctg} \frac{y}{x}, \operatorname{Arctg} \frac{\omega}{z}\right) . \\
& \cdot 4 \delta\left(x^{2}+y^{2}-z^{2}-\omega^{2}-1\right) .
\end{aligned}
$$

Inversely, if the distribution $P_{1}(x, y, z, \omega)$ is known, then it always contains the delta function

$P_{1}(x, y, z, \omega)=\tilde{P}(x, y, z, \omega) \cdot 4 \delta\left(x^{2}+y^{2}-z^{2}-\omega^{2}-1\right)$. and the distribution of $\rho, \varphi, \theta$ is given by the relation

$$
\begin{gathered}
P(\rho, \varphi, \theta)= \\
=\tilde{P}(\sqrt{1+\rho} \cos \varphi, \sqrt{1+\rho} \sin \varphi, \sqrt{\rho} \cos \theta, \sqrt{\rho} \sin \theta) .
\end{gathered}
$$

A transformation of the matrix $T$ under the change of the ideal leads is given by relations (17), (18), which are rather tremendous, but can be written in the compact form. As clear from the stated above, the case $k=\kappa$ is special for a situation in the forbidden band: it corresponds to the maximal transparency of interfaces (see Eq.4), while the phase $\psi=\theta-\varphi$ is localized near $\pi / 2$ for all $L$ (Sec.3). If the values of parameters for $k=\kappa$ are marked with a bar, then the relation (18) can be rewritten in the form

$$
x=\bar{x}, \quad y=\Delta_{2} \bar{y}-\Delta_{1} \bar{\omega}, \quad z=\bar{z}, \quad \omega=-\Delta_{1} \bar{y}+\Delta_{2} \bar{\omega},
$$

or inversely,

$$
\bar{x}=x, \quad \bar{y}=\Delta_{2} y+\Delta_{1} \omega, \quad \bar{z}=z, \quad \bar{\omega}=\Delta_{1} y+\Delta_{2} \omega,
$$

where $\Delta_{1}$ and $\Delta_{2}$ are defined in Eq.9. The elements $\bar{T}_{11}=\bar{x}+i \bar{y}$ and $\bar{T}_{12}=\bar{z}+i \bar{\omega}$ correspond to the momentum $\kappa$ and are determined by the internal properties of the system, while information on the ideal leads (i.e. the Fermi momentum $k$ ) contains in $\Delta_{1}$ and $\Delta_{2}$.

The case $k=\bar{k}$ is special for a situation in the allowed band (Fig.2,a). Marking by a bar the values of parameters, corresponding to this case and setting formally $\bar{k}=\kappa$, one can verify that the relation between $T_{i j}$ and $\bar{T}_{i j}$ is also given by Eq.64,65. If the distribution function $\bar{P}(\rho, \varphi, \theta)$ (determined by the internal properties of the system) is known for two indicated special situations, then (61) gives corresponding distribution $\bar{P}_{1}(\bar{x}, \bar{y}, \bar{z}, \bar{\omega})$, while the change of variables (65) gives $P_{1}(x, y, z, \omega)$, and then (63) gives the required distribution $P(\rho, \varphi, \theta)$, depending on the ideal leads:

$$
\begin{gathered}
P(\rho, \varphi, \theta)=\bar{P}\left(\rho^{\prime}, \varphi^{\prime}, \theta^{\prime}\right), \\
\rho^{\prime}=\rho \cos ^{2} \theta+\left(\Delta_{1} \sqrt{1+\rho} \sin \varphi+\Delta_{2} \sqrt{\rho} \sin \theta\right)^{2}, \\
\operatorname{tg} \varphi^{\prime}=\frac{\Delta_{2} \sqrt{1+\rho} \sin \varphi+\Delta_{1} \sqrt{\rho} \sin \theta}{\sqrt{1+\rho} \cos \varphi} \\
\operatorname{tg} \theta^{\prime}=\frac{\Delta_{1} \sqrt{1+\rho} \sin \varphi+\Delta_{2} \sqrt{\rho} \sin \theta}{\sqrt{\rho} \cos \theta}
\end{gathered}
$$

In the forbidden band we have for $k=\kappa$

$$
\bar{P}(\rho, \varphi, \theta)=\bar{P}(\rho) \delta(\varphi) \delta\left(\theta-\frac{\pi}{2}\right),
$$


where the delta functions should be broaden to the width of order $\epsilon$. If we neglect this widening, then

$$
P(\rho, \varphi, \theta)=\frac{1}{\Delta_{2}^{2}} \bar{P}\left(\frac{\rho}{\Delta_{2}^{2}}\right) \delta\left(\theta-\frac{\pi}{2}\right) \cdot \delta\left(\varphi+\arcsin \frac{\Delta_{1}}{\Delta_{2}} \sqrt{\frac{\rho}{1+\rho}}\right)
$$

and integration over $\varphi$ and $\theta$ gives the result (6), i.e. ideal leads do not change the form of $P(\rho)$ and only renormalize the absolute scale of conductance.

In the deep of the allowed band for $k=\bar{k}$ (Fig.2,a) the distribution $\bar{P}(\rho, \varphi, \theta)$ does not depend on the phase variables 4

$$
\bar{P}(\rho, \varphi, \theta)=\frac{1}{(2 \pi)^{2}} \bar{P}(\rho)=\frac{1}{(2 \pi)^{2} t} \exp \left(-\frac{\rho}{t}\right),
$$

and $\bar{P}(\rho)$ is determined by Eq.7 with $\gamma=0$. For the foreign leads (Fig.2,b) the distribution of $\rho$ is given by the integral

$$
P(\rho)=\frac{1}{(2 \pi)^{2} t} \int_{-\pi}^{\pi} d \varphi \int_{-\pi}^{\pi} d \theta \exp \left\{-\frac{\rho+S(\rho, \varphi, \theta)}{t}\right\},
$$

where

$$
S(\rho, \varphi, \theta)=\Delta_{1}^{2}(1+\rho) \sin ^{2} \varphi+2 \Delta_{1} \Delta_{2} \sqrt{(1+\rho) \rho} \sin \varphi \sin \theta+\Delta_{1}^{2} \rho \sin ^{2} \theta .
$$

Calculation of the integral (see Appendix 2) gives the following results.

In the case $\Delta_{1}^{2} \ll 1$, two regions are actual, $t \ll \Delta_{1}^{2}$ and $t \gg \Delta_{1}^{2}$; in the first of them

$$
P(\rho)=\left\{\begin{array}{cc}
\sqrt{\frac{1}{\pi \Delta_{1}^{2} t} \exp \left(-\frac{\rho}{2 t}\right),} & \rho \lesssim t \\
\frac{1}{\pi} \sqrt{\frac{1}{\rho\left(\rho_{c}-\rho\right)},} & t \lesssim \rho, \quad \rho_{c}-\rho \gtrsim\left(t \Delta_{1}^{2}\right)^{1 / 2} \\
\frac{1}{2 \pi^{2}} \sqrt{\frac{\pi}{2 \rho\left(t \Delta_{1}^{2}\right)^{1 / 2}} \Gamma(1 / 4),} & \left|\rho_{c}-\rho\right| \lesssim\left(t \Delta_{1}^{2}\right)^{1 / 2} \\
\frac{1}{\pi \sqrt{A B}} \exp \left(-\frac{S_{c}}{t}\right), & \rho-\rho_{c} \gtrsim\left(t \Delta_{1}^{2}\right)^{1 / 2}
\end{array},\right.
$$

where

$$
\begin{gathered}
S_{c}=\left(\Delta_{1} \sqrt{1+\rho}-\Delta_{2} \sqrt{\rho}\right)^{2} \Theta\left(\rho-\rho_{c}\right), \quad \rho_{c}=\Delta_{1}^{2}, \\
A=2 \Delta_{1} \sqrt{1+\rho}\left(\Delta_{2} \sqrt{\rho}-\Delta_{1} \sqrt{1+\rho}\right), \quad B=2 \Delta_{1} \sqrt{\rho}\left(\Delta_{2} \sqrt{1+\rho}-\Delta_{1} \sqrt{\rho}\right),
\end{gathered}
$$

and the main probability corresponds to the second result in (72). In the region $t \gg \Delta_{1}^{2}$ one has

$$
P(\rho)=\left\{\begin{array}{cc}
\frac{1}{t} \exp \left(-\frac{\rho}{t}\right)\left[1-\frac{\Delta_{1}^{2}}{2 t}-\frac{\Delta_{1}^{2} \rho}{t}+\frac{\Delta_{1}^{2} \Delta_{2}^{2} \rho(1+\rho)}{2 t^{2}}\right], & \rho(1+\rho) \lesssim t^{2} / \Delta_{1}^{2} \\
\frac{1}{\pi \sqrt{A B}} \exp \left(-\frac{S_{c}}{t}\right), & \rho(1+\rho) \gtrsim t^{2} / \Delta_{1}^{2}
\end{array},\right.
$$

and the normalization integral is determined by the first asymptotics. Evolution of the distribution for $\Delta_{1}^{2} \ll 1$ is shown in Fig.5,a. At small $t$ it reduces to the smearing of singularities of the distribution (8),

\footnotetext{
${ }^{4}$ For this, strictly speaking, one should average (32) over the $n$ variations of order $1 / \delta$.
} 
while in the limit of large $t$ the value $P(\rho)$ at $\rho=0$ tends to one of the distribution $\bar{P}(\rho)$ and the whole form of the distribution is close to the latter.

In the case $\Delta_{1}^{2} \gg 1$, three regions are actual, $t \ll 1,1 \ll t \ll \Delta_{1}^{2}$ and $t \gg \Delta_{1}^{2}$. In the first of them one has results

$$
P(\rho)=\left\{\begin{array}{cc}
\sqrt{\frac{1}{\pi \Delta_{1}^{2} t} \exp \left(-\frac{\rho}{2 t}\right),} & \rho \lesssim t \\
\frac{1}{\pi} \sqrt{\frac{1}{\rho\left(\rho_{c}-\rho\right)},} & t \lesssim \rho, \quad \rho_{c}-\rho \gtrsim\left(t \Delta_{1}^{2} \rho\right)^{1 / 2} \\
\frac{1}{2 \pi^{2}} \sqrt{\frac{\pi}{2 \rho\left(t \Delta_{1}^{2} \rho\right)^{1 / 2}} \Gamma(1 / 4),} & \left|\rho_{c}-\rho\right| \lesssim\left(t \Delta_{1}^{2} \rho\right)^{1 / 2} \\
\frac{1}{\pi \sqrt{A B}} \exp \left(-\frac{S_{c}}{t}\right), & \rho-\rho_{c} \gtrsim\left(t \Delta_{1}^{2} \rho\right)^{1 / 2}
\end{array},\right.
$$

which are analogous to (72) and correspond to smearing of singularities of the distribution (8); the main probability corresponds to the second asymptotics. In the region $1 \ll t \ll \Delta_{1}^{2}$ we obtain

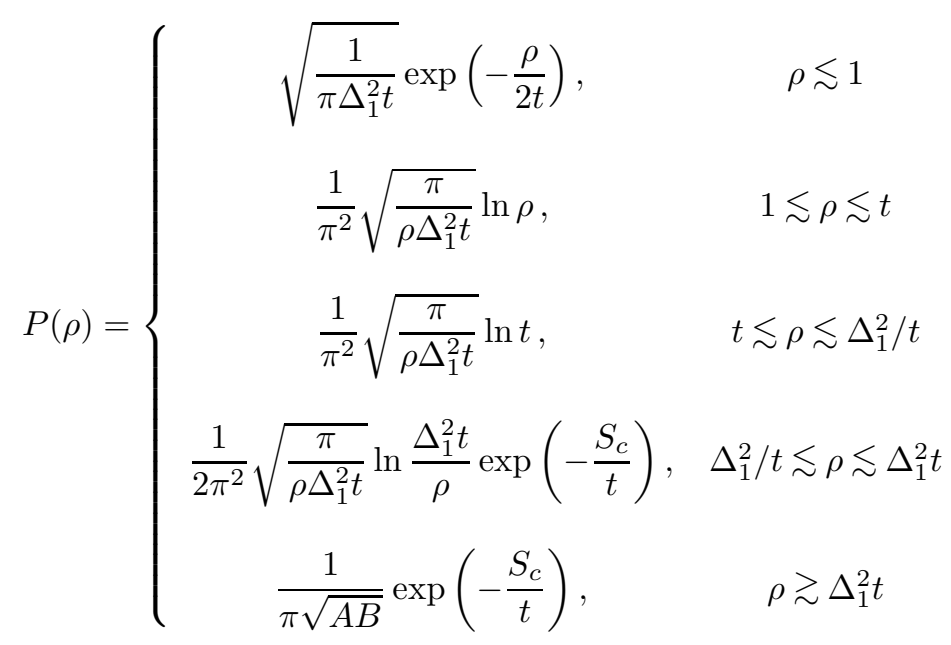

while in the region $t \gg \Delta_{1}^{2}$

$$
P(\rho)=\left\{\begin{array}{cc}
\frac{1}{t} \exp \left(-\frac{\rho}{t}\right)\left[1-\frac{\Delta_{1}^{2}}{2 t}-\frac{\Delta_{1}^{2} \rho}{t}+\frac{\Delta_{1}^{2} \Delta_{2}^{2} \rho(1+\rho)}{2 t^{2}}\right], & \rho \lesssim t / \Delta_{1}^{2} \\
\frac{1}{\pi^{2}} \sqrt{\frac{\pi}{\rho \Delta_{1}^{2} t} \ln \frac{\Delta_{1}^{2} \rho}{t},} & t / \Delta_{1}^{2} \lesssim \rho \lesssim t \\
\frac{1}{2 \pi^{2}} \sqrt{\frac{\pi}{\rho \Delta_{1}^{2} t} \ln \frac{\Delta_{1}^{2} t}{\rho} \exp \left(-\frac{S_{c}}{t}\right),} & t \lesssim \rho \lesssim \Delta_{1}^{2} t \\
\frac{1}{\pi \sqrt{A B}} \exp \left(-\frac{S_{c}}{t}\right), & \rho \gtrsim \Delta_{1}^{2} t
\end{array}\right.
$$

In both cases the main probability is related with two last asymptotic results. The characteristic feature of (76) and (77) is existence of the quick exponent $(\exp (-\rho / 2 t)$ or $\exp (-\rho / t))$ for small $\rho$ and the slow 
exponent $\exp \left(-\rho / \Delta_{1}^{2} t\right)$ for large $\rho$, while the power law behavior $P(\rho) \propto \rho^{-1 / 2}$ is valid for intermediate $\rho$, apart of the logarithmic corrections. Evolution of the distribution for $\Delta_{1}^{2} \gg 1$ is shown in Fig.5,b.

In the above discussion we had in mind that results depend on $\left|\Delta_{1}\right|$ (which can be tested by the change $\varphi \rightarrow \varphi+\pi$ in Eqs.70,71) and $\Delta_{1}$ can be consider as positive without the loss of generality. In addition, we assumed that the distribution (69) is given axiomatically and did not discuss its conditions of applicability: it allows to understand better, how $P(\rho)$ is transformed due to ideal leads. In fact, the distribution (69) corresponds to solution of equation (2) only for $\rho \lesssim 1$, which restricts the physical actuality of (76), (77) by the condition $\rho \lesssim \Delta_{1}^{2}$.

In the log-normal regime, arising for $t \gg 1$, one should take $\bar{P}(\rho)$ in the form (5) with $\gamma=0$. Since the typical values of $\rho$ are large, we can set $\rho^{\prime}=$ $\rho K(\varphi, \theta)$ in Eq.66, where

$$
K(\varphi, \theta)=\cos ^{2} \theta+\left(\Delta_{1} \sin \varphi+\Delta_{2} \sin \theta\right)^{2} .
$$

Substitution to (5), (66), (69) gives

$$
\begin{gathered}
P(\rho, \varphi, \theta)=\frac{1}{(2 \pi)^{2}} \frac{1}{\rho K \sqrt{4 \pi t}} \exp \left\{-\frac{(\ln K \rho-t)^{2}}{4 t}\right\} \approx \\
\approx \frac{1}{(2 \pi)^{2}} \frac{1}{\rho \sqrt{4 \pi t}} \exp \left\{-\frac{(\ln \rho-t)^{2}}{4 t}\right\} . \\
\cdot\left[\frac{1}{K}-\frac{2(\ln \rho-t) \ln K}{4 t K}\right], \quad \text { (79) }
\end{gathered}
$$

where expansion in $1 / t$ is produced, using the fact that $(\ln \rho-t) \sim \sqrt{t}$ for the bulk of the distribution. Integrating over $\varphi$ and $\theta$, we set

$$
\begin{aligned}
& \int_{-\pi}^{\pi} \frac{d \varphi}{2 \pi} \int_{-\pi}^{\pi} \frac{d \theta}{2 \pi} \frac{1}{K(\varphi, \theta)} \equiv \frac{1}{K_{0}}, \\
& \int_{-\pi}^{\pi} \frac{d \varphi}{2 \pi} \int_{-\pi}^{\pi} \frac{d \theta}{2 \pi} \frac{\ln K(\varphi, \theta)}{K(\varphi, \theta)} \equiv \frac{\ln K_{1}}{K_{0}} .
\end{aligned}
$$

Then conservation of probability requires the condition $K_{0}=1$, under which $P(\rho)$ can be written in the form

$$
P(\rho)=\frac{1}{(2 \pi)^{2}} \frac{1}{\rho \sqrt{4 \pi t}} \exp \left\{-\frac{\left(\ln K_{1} \rho-t\right)^{2}}{4 t}\right\} .
$$

The equality $K_{0}=1$ is indeed valid, as one can verify by a direct calculation of the integral. We see



Figure 8: Parameter $K_{1}$ as function of $\Delta_{1}$.

that the ideal leads do not change the parameters of the log-normal distribution and only renormalize the absolute scale of $\rho$, which is determined by the parameter $K_{1}$ (Fig.8):

$$
K_{1}=\left\{\begin{array}{ll}
1-\Delta_{1}^{2} / 2, & \Delta_{1} \ll 1 \\
\text { const } / \Delta_{1}^{2}, & \Delta_{1}^{2} \gg 1
\end{array},\right.
$$

where the constant is numerically close to 4 .

In the critical region for sufficiently small $n$ we can begin with Eq.38, which determines the distribution $\bar{P}(\rho, \varphi, \theta)$ in the form (39). A transformation of variables (64) results in the change $S \rightarrow S\left(\Delta_{2}-\Delta_{1}\right)$ in (38),(39) and renormalization (6) of the absolute scale of conductance with $A=1 /\left(\Delta_{2}-\Delta_{1}\right)^{2}$.

\section{Evolution equation for $P(\rho)$ in the presence of the ideal leads}

According to Sec.3, the transfer matrix $T$ for a succession of point scatterers and the "natural" ideal leads (Fig.2,a) has a form (22); the corresponding parameters $\epsilon_{s}$ will be denoted as $\bar{\epsilon}_{s}$. In the presence of foreign leads (Fig.2,b) the system is described by the transfer matrix $\tilde{T}=T_{a} T T_{b}$. Inserting the product $T_{b} T_{a}=1$ between each two multipliers of (22), one has

$$
\tilde{T}^{(n)}=\tilde{T}_{\epsilon_{0}} \tilde{T}_{\delta_{1}} \tilde{T}_{\epsilon_{1}} \tilde{T}_{\delta_{2}} \tilde{T}_{\epsilon_{2}} \ldots \tilde{T}_{\delta_{n}} \tilde{T}_{\epsilon_{n}},
$$

where

$$
\tilde{T}_{\epsilon_{s}}=T_{a} T_{\epsilon_{s}} T_{b}, \quad \tilde{T}_{\delta}=T_{a} T_{\delta} T_{b} .
$$

In the allowed band the difference between $\tilde{T}_{\epsilon_{s}}$ and $T_{\epsilon_{s}}$ is reduced to renormalization $\bar{\epsilon}_{s} \rightarrow \epsilon_{s}=\bar{\epsilon}_{s} \bar{k} / k$, which has a simple physical sense. Let represent 
the initial system (Fig.9,a) as a succession of similar scatterers (Fig.9,b), creating the potential well for each point scatterer with the width tending to zero. Then the parameter $\bar{\epsilon}_{s}=V_{s} / 2 \bar{k} a_{0}$, defined for the Fermi momentum $\bar{k}$ of the system, is replaced by the parameter $\epsilon_{s}=V_{s} / 2 k a_{0}$, containing the Fermi momentum $k$ in the ideal leads. As for $\tilde{T}_{\delta}$, it is the transfer matrix of the potential barrier, separating two point scatterers in Fig.9,b:

$$
\begin{gathered}
\tilde{T}_{\delta}=\left(\begin{array}{cc}
\cos \delta-i \Delta_{2} \sin \delta & i \Delta_{1} \sin \delta \\
-i \Delta_{1} \sin \delta & \cos \delta+i \Delta_{2} \sin \delta
\end{array}\right) \approx \\
\approx\left(\begin{array}{cc}
1-i \Delta_{2} \delta & i \Delta_{1} \delta \\
-i \Delta_{1} \delta & 1+i \Delta_{2} \delta
\end{array}\right)
\end{gathered}
$$

where $\Delta_{1}$ and $\Delta_{2}$ are determined by Eq.9, if one set $\bar{k}=\kappa$. The situation in the forbidden band (Fig.2,c) differs only by the fact, that the height of barriers in Fig.9,b prevails the Fermi level, while $\tilde{T}_{\delta}$ is obtained from Eq. 85 by the replacement of $\bar{k}$ by $i \kappa$

$$
\begin{gathered}
\tilde{T}_{\delta}=\left(\begin{array}{cc}
\operatorname{ch} \delta-i \Delta_{1} \operatorname{sh} \delta & i \Delta_{2} \operatorname{sh} \delta \\
-i \Delta_{2} \operatorname{sh} \delta & \operatorname{ch} \delta+i \Delta_{1} \operatorname{sh} \delta
\end{array}\right) \approx \\
\approx\left(\begin{array}{cc}
1-i \Delta_{1} \delta & i \Delta_{2} \delta \\
-i \Delta_{2} \delta & 1+i \Delta_{1} \delta
\end{array}\right)
\end{gathered}
$$

Let describe the potential barrier by the transfer matrix of the general form

$$
\tilde{T}_{\delta}=\left(\begin{array}{ll}
A & B \\
B^{*} & A^{*}
\end{array}\right)=\left(\begin{array}{cc}
\sqrt{1+\Delta^{2}} \mathrm{e}^{i \alpha} & \Delta \mathrm{e}^{-i \beta} \\
\Delta \mathrm{e}^{i \beta} & \sqrt{1+\Delta^{2}} \mathrm{e}^{-i \alpha}
\end{array}\right)
$$

close to the unit one $(\alpha, \Delta \ll 1)$; it has eigenvalues $\mathrm{e}^{ \pm \delta}$, where $\delta^{2}=\Delta^{2}-\alpha^{2}$. According to $(83), \tilde{T}^{(n)}$ can be expressed as $\tilde{T}^{(n-1)}$ multiplied by $\tilde{T}_{\delta} \tilde{T}_{\epsilon_{n}}$. Accepting $\tilde{T}^{(n-1)}$ in the form (12), one has

$$
\tilde{T}_{12}^{(n)}=\sqrt{1+\rho} \mathrm{e}^{i \varphi}(B+i \epsilon C)+\sqrt{\rho} \mathrm{e}^{i \theta}\left(A^{*}-i \epsilon C^{*}\right),
$$

where $C=B-A$. Squaring the modulus of (88), we have the value of $\tilde{\rho}$, corresponding to $\tilde{T}^{(n)}$

$$
\tilde{\rho}=\rho+D \sqrt{\rho(1+\rho)}+\epsilon^{2}(1+2 \rho),
$$

where

$$
\begin{gathered}
D=2 \Delta \cos (\psi+\beta)-2 \epsilon \sin \psi-2 \epsilon^{2} \cos \psi, \\
\psi=\theta-\varphi,
\end{gathered}
$$

and we retained the terms of the first order in $\delta$ and the second order in $\epsilon$. Expression (89) is analogous

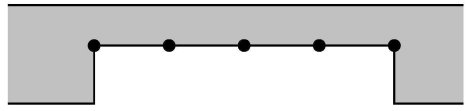

(a)

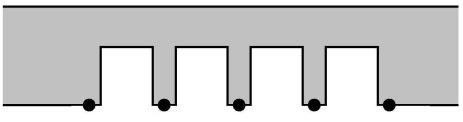

(b)

Figure 9: The physical sense of the $\epsilon_{s}$ renormalization consists in representation of the initial system (a) as a succession of similar scatterers (b). In the latter case the potential well is created for each point scatterer, whose width tends to zero.

to Eq.(A.2) of the paper [12] and subsequent calculations follow to Appendix $A$ of this paper. As a result, we obtain Eq.3 with parameters (we set $a_{0}=1$ )

$$
\tilde{\alpha}=\frac{1}{2} \overline{D^{2}}, \quad \gamma_{1} \tilde{\alpha}=\epsilon^{2}-\frac{1}{2} \overline{D^{2}}, \quad \gamma_{2} \tilde{\alpha}=\frac{1}{2} \bar{D},
$$

and substitution of (90) gives

$$
\begin{gathered}
\tilde{\alpha}=2 \epsilon^{2} \overline{\sin ^{2} \psi}, \quad \gamma_{1} \tilde{\alpha}=\epsilon^{2}\left(1-\overline{2 \sin ^{2} \psi}\right), \\
\gamma_{2} \tilde{\alpha}=\Delta \overline{\cos (\psi+\beta)}-\epsilon^{2} \overline{\cos \psi}
\end{gathered}
$$

where $\beta=-\pi / 2$ in the actual case; another values of $\beta$ occur in the presence of the $\delta$-function potential on interfaces.

Equation (3) with parameters (93) allows to analyze the special situations with $\epsilon=\bar{\epsilon}$, actual for Sec.6. In the deep of the allowed band and $k=\bar{k}$ we have $\Delta_{1}=0$ and $\Delta=0$ (see $\left.(85),(87)\right)$; in the case of the uniform distribution for $\psi$, Eq.93 gives $\tilde{\alpha}=\epsilon^{2}, \gamma_{1}=0, \gamma_{2}=0$, in agreement with the results of Secs.4,5. Hence, the uniform distribution for $\psi$ obtained in Sec.3 for $n \lesssim 1 / \epsilon^{2}$ retains also for $n \gtrsim 1 / \epsilon^{2}$. In the deep of the forbidden band and for $k=\kappa$ one has $\Delta_{1}=0$ and $\Delta=\delta$ (see (86),(87)); the results $\tilde{\alpha}=2 \epsilon^{2}, \gamma \tilde{\alpha}=\delta$, obtained in Secs.4,5, agree with (93), if localization of $\psi$ near $\pi / 2$ is accepted; such localization was established in Sec.3 for $n \lesssim 1 / \epsilon^{2}$ and retains for $n \gtrsim 1 / \epsilon^{2}$. In the critical region $\Delta_{1} \approx \Delta_{2}$ and $\Delta \sim \delta$, so the results $\tilde{\alpha} \sim\left(\epsilon^{2} \delta^{2}\right)^{1 / 3}$, $\gamma=\gamma_{1}+\gamma_{2} \sim 1$ of Sec.4,5 agree with (93) in the case of localization of $\psi$ in the region of small values $\sim\left(\delta / \epsilon^{2}\right)^{1 / 3}$, in accordance with the analysis of Sec.3.

\section{Evolution of the mean value of $\rho$.}


Evolution of $\langle\rho\rangle$ allows a complete description and illustrates the influence of the ideal leads for the arbitrary system length $L$. The relation $\tilde{T}^{(n)}=\tilde{T}^{(n-1)} \tilde{T}_{\delta} \tilde{T}_{\epsilon_{n}}$ has the following form in terms of the matrix elements

$$
\tilde{T}_{11}^{(n)}=\left(1+i \alpha-i \epsilon_{n}\right) \tilde{T}_{11}^{(n-1)}+\left(-i \Delta+i \epsilon_{n}\right) \tilde{T}_{12}^{(n-1)}, \quad \tilde{T}_{12}^{(n)}=\left(i \Delta-i \epsilon_{n}\right) \tilde{T}_{11}^{(n-1)}+\left(1-i \alpha+i \epsilon_{n}\right) \tilde{T}_{12}^{(n-1)},
$$

where we linearized in $\alpha, \Delta$ and set $\beta=-\pi / 2$. Introducing notations for the second moments

$$
z_{1}^{(n)}=\left\langle\left|\tilde{T}_{11}^{(n)}\right|^{2}\right\rangle, \quad z_{2}^{(n)}=\left\langle\tilde{T}_{11}^{(n)} \tilde{T}_{12}^{(n) *}\right\rangle, \quad z_{3}^{(n)}=\left\langle\tilde{T}_{11}^{(n) *} \tilde{T}_{12}^{(n)}\right\rangle, \quad z_{4}^{(n)}=\left\langle\left|\tilde{T}_{12}^{(n)}\right|^{2}\right\rangle,
$$

we have the system of difference equations

$$
\left(\begin{array}{c}
z_{1}^{(n)} \\
z_{2}^{(n)} \\
z_{3}^{(n)} \\
z_{4}^{(n)}
\end{array}\right)=\left(\begin{array}{cccc}
1+\epsilon^{2} & i \Delta-\epsilon^{2} & -i \Delta-\epsilon^{2} & \epsilon^{2} \\
-i \Delta-\epsilon^{2} & 1+2 i \alpha-\epsilon^{2} & -\epsilon^{2} & -i \Delta+\epsilon^{2} \\
i \Delta-\epsilon^{2} & -\epsilon^{2} & 1-2 i \alpha-\epsilon^{2} & i \Delta+\epsilon^{2} \\
\epsilon^{2} & i \Delta-\epsilon^{2} & -i \Delta-\epsilon^{2} & 1+\epsilon^{2}
\end{array}\right)\left(\begin{array}{c}
z_{1}^{(n-1)} \\
z_{2}^{(n-1)} \\
z_{3}^{(n-1)} \\
z_{4}^{(n-1)}
\end{array}\right)
$$

whose solution is exponential, $z_{i}^{(n)} \sim \lambda^{n}$, and $\lambda$ is an eigenvalue of the matrix; substitution $\lambda=1+x$ leads to the equation

$$
x\left[x\left(x^{2}+4 \alpha^{2}-4 \Delta^{2}\right)-8 \epsilon^{2}(\Delta-\alpha)^{2}\right]=0,
$$

which has the solution $x=0$ and three nontrivial roots. The latter coincide with roots of Eq.44 and do not depend on the ideal leads, since $\delta^{2}=\Delta^{2}-\alpha^{2}$ and

$$
8 \epsilon^{2}(\Delta-\alpha)^{2}=8 \epsilon^{2} \delta^{2}\left(\Delta_{2}-\Delta_{1}\right)^{2}=8 \bar{\epsilon}^{2} \delta^{2}=2 W^{2} .
$$

Finding the eigenvectors of matrix (96), we have the general solution for $z_{i}^{(n)}$

$$
\left(\begin{array}{c}
z_{1}^{(n)} \\
z_{2}^{(n)} \\
z_{3}^{(n)} \\
z_{4}^{(n)}
\end{array}\right)=C_{0}\left(\begin{array}{c}
-1 \\
0 \\
0 \\
1
\end{array}\right)+\sum_{i=1}^{3} C_{i}\left(\begin{array}{c}
1 \\
e_{2}\left(x_{i}\right) \\
e_{3}\left(x_{i}\right) \\
1
\end{array}\right) \exp \left(x_{i} n\right)
$$

where $x_{1}, x_{2}, x_{3}$ are nontrivial roots of $(97)$, and

$$
\begin{gathered}
e_{2}(x)=\frac{\mathcal{A} x+\mathcal{B}}{p(x)}, \quad e_{3}(x)=\frac{\mathcal{A}^{*} x+\mathcal{B}^{*}}{p(x)}, \\
\mathcal{A}=2 \epsilon^{2}-2 i \Delta, \quad \mathcal{B}=4 \alpha \Delta+4 i \epsilon^{2}(\alpha-\Delta), \\
p(x)=x^{2}+2 \epsilon^{2} x+4 \alpha^{2} .
\end{gathered}
$$

Choosing the unit transfer matrix as the initial condition, we have $z_{1}^{(0)}=1, z_{2}^{(0)}=z_{3}^{(0)}=z_{4}^{(0)}=0$, which allows to establish the coefficients $C_{i}$. Since $z_{4}^{(n)}$ gives immediately $\langle\rho\rangle$, then

$$
\langle\rho\rangle=-\frac{1}{2}+\frac{\left(x_{2}-x_{3}\right) p\left(x_{1}\right)}{2 Q} \mathrm{e}^{x_{1} n}-\frac{\left(x_{1}-x_{3}\right) p\left(x_{2}\right)}{2 Q} \mathrm{e}^{x_{2} n}+\frac{\left(x_{1}-x_{2}\right) p\left(x_{3}\right)}{2 Q} \mathrm{e}^{x_{3} n},
$$

where

$$
Q=x_{1}^{2}\left(x_{2}-x_{3}\right)-x_{2}^{2}\left(x_{1}-x_{3}\right)+x_{3}^{2}\left(x_{1}-x_{2}\right) .
$$

The result (101) has the general character, since no approximations were made in its derivation. For large $n$ one of the exponents is dominated, and a situation is multiplicative (Sec.1). For small $n$ the exponents can be extended in the series, recovering the Ohmic regime $\rho \propto n$. 
In the localized regime $\left(\delta \gg \bar{\epsilon}^{2}\right)$, the nontrivial roots of Eq.97 have a form

$$
x_{1}=2 \delta+\bar{\epsilon}^{2}, \quad x_{2}=-2 \bar{\epsilon}^{2}, \quad x_{3}=-2 \delta+\bar{\epsilon}^{2},
$$

and

$$
\langle\rho\rangle=-\frac{1}{2}+\frac{\Delta_{2}^{2}}{4}\left(\mathrm{e}^{x_{1} n}+\mathrm{e}^{x_{3} n}\right)-\frac{\Delta_{1}^{2}}{2} \mathrm{e}^{x_{2} n} .
$$

For large $n$ the term $\mathrm{e}^{x_{1} n}$ is dominated, confirming renormalization of $\rho$ by the factor $\Delta_{2}^{2}$, indicated in Eq.6; the change of the $n$ origin (see the end of Sec.5) is essential only for $n \lesssim 1 / \delta$.

In the metallic regime one makes the change $\delta \rightarrow$ $i \delta, \epsilon \rightarrow-i \epsilon$ and considers the limit $\delta \gg \bar{\epsilon}^{2}$; then

$$
x_{1}=2 \bar{\epsilon}^{2}, \quad x_{2}=-\bar{\epsilon}^{2}+2 i \delta, \quad x_{3}=-\bar{\epsilon}^{2}-2 i \delta,
$$

and

$$
\langle\rho\rangle=-\frac{1}{2}+\frac{\Delta_{2}^{2}}{2} \mathrm{e}^{2 \bar{\epsilon}^{2} n}-\frac{\Delta_{1}^{2}}{2} \mathrm{e}^{-\bar{\epsilon}^{2} n} \cos 2 n \delta,
$$

which for the "natural" leads (when $\Delta_{1}=0$ ) gives the well-known result [1, 2, 15, 16, 17.

$$
\langle\rho\rangle=\frac{1}{2}\left(\mathrm{e}^{2 \bar{\epsilon}^{2} n}-1\right) .
$$

For finite $\Delta_{1}$, the oscillations arise whose period is determined by the de Broglie wavelength (since $2 n \delta=2 \bar{k} L)$. Their origin is clear, since for $\bar{\epsilon}=0$ the Landauer resistance $\rho$ is determined by the transfer matrix (85) of the potential barrier,

$$
\rho=\Delta_{1}^{2} \sin ^{2} \bar{k} L,
$$

which becomes transparent, if the system length corresponds to the semi-integer number of the de Broglie wavelengths (analogously to blooming in optics). For finite $\bar{\epsilon}$, the oscillations become attenuating, but remain completely observable: the mean value of $\rho$ is representative in the metallic regime, since its fluctuations are relatively small.

In the critical region $\left(\delta \ll \bar{\epsilon}^{2}\right)$ one has

$x_{1}=2\left(\bar{\epsilon}^{2} \delta^{2}\right)^{1 / 3}, \quad x_{2}=x_{1} \mathrm{e}^{2 \pi i / 3}, \quad x_{3}=x_{1} \mathrm{e}^{-2 \pi i / 3}$,

and

$$
\begin{aligned}
& \langle\rho\rangle=\frac{1}{6}\left[\mathrm{e}^{x_{1} n}+2 \mathrm{e}^{-x_{1} n / 2} \cos \left(\frac{\sqrt{3}}{2} x_{1} n\right)-3\right]+ \\
& +\frac{\epsilon^{2}}{3 x_{1}}\left[\mathrm{e}^{x_{1} n}-2 \mathrm{e}^{-x_{1} n / 2} \cos \left(\frac{\sqrt{3}}{2} x_{1} n+\frac{\pi}{3}\right)\right]+
\end{aligned}
$$

$$
+\frac{2 \alpha^{2}}{3 x_{1}^{2}}\left[\mathrm{e}^{x_{1} n}-2 \mathrm{e}^{-x_{1} n / 2} \cos \left(\frac{\sqrt{3}}{2} x_{1} n-\frac{\pi}{3}\right)\right] .
$$

If the critical region is approached from the allowed band, then the "natural" ideal leads can be used (Fig.2,a), when $\epsilon=\bar{\epsilon}$ and the second term is dominated in Eq.110. Expansion in $x_{1} n$ gives $\langle\rho\rangle=\epsilon^{2} n$ in agreement with the transfer matrix (38), and establishes its range of applicability as $n \lesssim 1 / x_{1}$.

The result (110) remains finite in the limit $\bar{k} \rightarrow 0$, when

$$
\begin{gathered}
\frac{\epsilon^{2}}{3 x_{1}}=\frac{\left(2 W^{2}\right)^{2 / 3}}{24 k^{2} a_{0}^{2}}, \quad \frac{2 \alpha^{2}}{3 x_{1}^{2}}=\frac{2 k^{2} a_{0}^{2}}{3\left(2 W^{2}\right)^{2 / 3}}, \\
x_{1}=\left(2 W^{2}\right)^{1 / 3} .
\end{gathered}
$$

Analogously to (106), the attenuating oscillations take place, whose amplitude depends essentially on the Fermi momentum $k$ in the ideal leads: it is of the order of unity for $k a_{0} \sim W^{2 / 3}$ (when three terms in Eq.110 are of the same order), but increases for small $k$ (when the second term is dominated), as well as for large $k$ (when the third term is prevailed). The period of the oscillations is determined by the amplitude of the random potential, while the phase shift changes from $\pi / 3$ till $-\pi / 3$ when $k$ is increased.

In fact, the observable picture is more complicated. In the critical region fluctuations of $\rho$ are large, and the form of the distribution function essentially depends on the several first moments 5 . Meanwhile, the higher moments of $\rho$ are also oscillating with the period of the same order: it is related with the complex roots of Eq.48 and analogous equations for higher moments 6. As a result, the complicated interference of incommensurate oscillations occurs, so the resistance $\rho$ of the specific sample undergoes aperiodic oscillations. Such oscillations were

\footnotetext{
${ }^{5}$ Let us remind that the Fourier transform of $P(\rho)$ gives the characteristic function $F(t)=\left\langle e^{i \rho t}\right\rangle$, which is the generating function of moments, $F(t)=\sum_{n=0}^{\infty}(i t)^{n}\left\langle\rho^{n}\right\rangle / n$ !. If all moments of the distribution are known, then one can construct $F(t)$, while $P(\rho)$ is given by the inverse Fourier transform.

${ }^{6}$ Comparison of (44) and (48) shows that the periods of oscillations for $\langle\rho\rangle$ and $\left\langle\rho^{2}\right\rangle$ differ by a factor $21^{1 / 3}$. As clear from Sec.4 (see Footnote 3 ), the right hand side of the equation for $x$ may contain only combinations $\delta^{2 n} \epsilon^{2 m}$ with $n \geq m$, of which only $\delta^{2 n} \epsilon^{2 n} \sim W^{2 n}$ remain finite in the $\delta \rightarrow 0$ limit. Since for $x \sim \delta \sim \epsilon^{2}$ all terms of the equation have the same order of magnitude, the exponent of growth $x$ for $\left\langle\rho^{n}\right\rangle$ at $\delta=0$ satisfies the equation $x^{2 n+1}=c_{1} \delta^{2} \epsilon^{2} x^{2 n-2}+c_{2} \delta^{4} \epsilon^{4} x^{2 n-5}+\ldots$, whose nontrivial roots are of order $\left(\delta^{2} \epsilon^{2}\right)^{1 / 3}$ independently of $n$.
} 


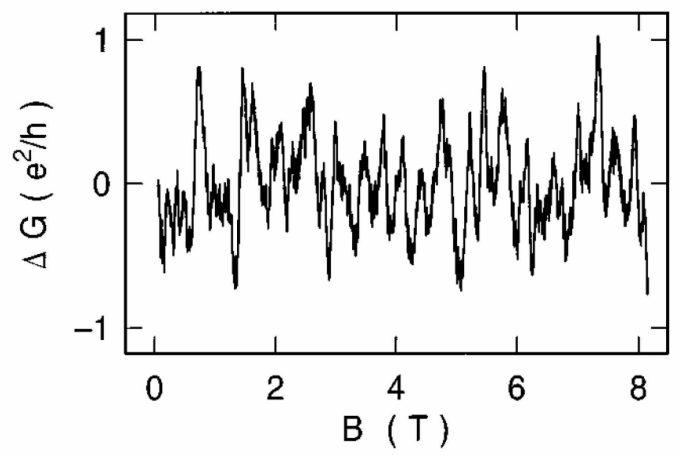

Figure 10: The conductance $G(B)$ of the thin Au wire 34 in the magnetic field $B$ undergoes the "universal" fluctuations of order $e^{2} / h$ due to a change of the impurity configuration 2526 . Fluctuations of $G(B)$ and $G(B+\Delta B)$ are statistically independent, if $\Delta B$ exceeds a certain characteristic scale [25] 26]. In spite of the random character, the fluctuation picture is completely reproducible and reflects the specific realization of the random potential ("magnetic fingerprints").

observed in the magneto-resistance of thin wires 34 (Fig.10) and have the close relation to the above discussion: the magnetic field, perpendicular to a wire, creates the quadratic potential along it, which effectively restricts the length of the system; so variation of the magnetic field is analogous to the change of $L$. In principle, Fig.10 is explained by the theoretical results of the papers [25, 26], but we are unaware on attempts of description of the oscillations themselves.

Fig.11 shows the experimental results of the paper [35], which demonstrate a possibility of observation of the entire distribution function of $\rho$ or $G$, as well as its moments. Independent impurity configurations in the thin wire of Si-doped GaAs were created by the periodic warming till the room temperature, and then averaging over 50 configurations were made. The results for the first two moments of $G$ indicate that the distribution function is not stationary, but undergoes systematic variations, in agreement with the above arguments.

\section{Conclusion}

The massive ideal leads which should be introduced for the correct definition of the conductance of finite systems have the essential influence on the properties of the given sample. In the present paper it was demonstrated on the simplest example of $1 \mathrm{D}$ systems. In the log-normal regime, this influence is reduced to the change of the absolute scale

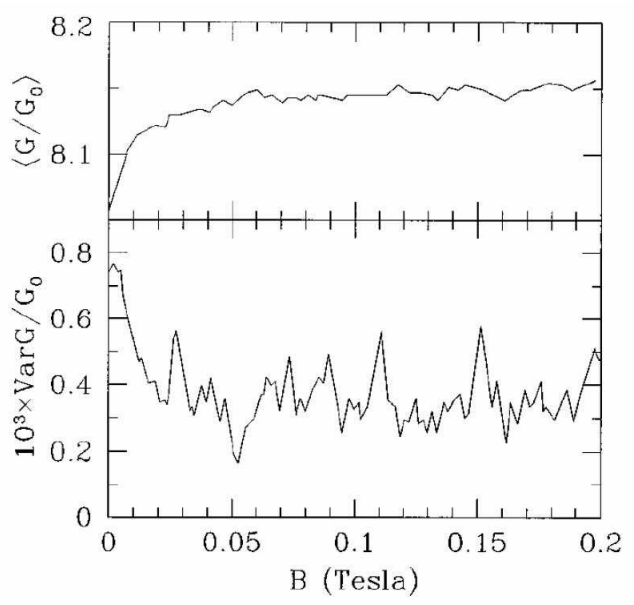

Figure 11: Evolution of the first two moments of the conductance $G$ of the thin Si-doped GaAs wire under the change of the magnetic field 35.

of conductance, but generally changes the whole distribution function. Under the change of the system length $L$, the system resistance may undergo the periodic or aperiodic oscillations. Variation of the Fermi level induces the qualitative changes in the conductance distribution, resembling the smoothed Anderson transition.

The Economou-Soukoulis definition of conductance [3] refers to the composite system "sample+ideal leads", while its relation to the system under consideration remains the open question. For its solution, the introduction of semi-transparent boundaries between the system and the ideal leads was suggested in [11. In the limit of the weakly transparent boundaries, one has the universal equations, independent of the way how the contact resistance of the reservoir is excluded [10 (since all formulas of the Landauer type [2, [5, 6, [8, 9] reduces to the variant by Economou-Soukoulis [3, 4]), which can be then extrapolated to transparency of order unity. Such definition refers surely to the given sample and provides the infinite conductance for the ideal system. However, the absolute scale of conductance is defined only in the order of magnitude; this point is not very significant (only the ratio of conductances has a physical sense), but creates certain difficulties in comparison with other results. As clear from the present paper, the absolute scale of conductance depends on the ideal leads, and in any case is a conditional quantity. To avoid its uncertainty, one should give information on the properties of leads: 
for example, one can accept the "Gold Standard" and recalculate all results to the leads made of gold.

According to Eq.2, the conductance distribution of an $1 \mathrm{D}$ system is determined by two parameters $\tilde{\alpha} L$ and $\gamma$. Such two parameter description is the natural consequence of the one-parameter scaling hypothesis 36, according to which the properties of a $d$-dimensional cubical system of size $L$ are completely determined by the ratio $L / \xi$, where $\xi$ is the correlation length. Composing the quasi-1D system of size $L^{d-1} L_{z}$ from the cubical blocks of size $L$, one comes to conclusion that its conductance depends on the properties of one block $(L / \xi)$ and a number of blocks $\left(L_{z} / L\right)$. For $L=a_{0}$ the quasi-1D system becomes strictly onedimensional, but is also described by two parameters. One-parameter scaling in $1 \mathrm{D}$ systems is realized in the deep of the allowed band (when equation (1) is valid) and approximately retains in the critical region7; its violation in the forbidden band is rather natural due to violation of the condition $\xi \gg a_{0}$. Necessity of the two-parameter description of 1D systems was discussed in the paper [37] (see also [21]-24])) and recently it was actively used for description of the conductance distribution near the Anderson transition $[12,38$ in the framework of the Shapiro scheme [18].

Evolution of the distribution function $P(\rho)$, as well as its moments, can be studied experimentally in the spirit of the paper [35], where different impurity configurations in the given sample were created by its warming to sufficiently high temperature.

\section{Appendix 1. Evolution of moments for the coordinate transfer matrix}

For the coordinate transfer matrix the following evolution equations are valid

$$
z_{n+1}=\left(E-V_{n}\right) z_{n}+y_{n}, \quad y_{n+1}=-z_{n},
$$

where $z_{n}=\tau_{12}^{(n-1)}, y_{n}=\tau_{11}^{(n-1)}$ with the initial conditions $z_{1}=0, y_{1}=1$, or $z_{n}=\tau_{22}^{(n-1)}, y_{n}=\tau_{21}^{(n-1)}$ with the initial conditions $z_{1}=1, y_{1}=0$. For the second moments one has

$$
\left(\frac{\overline{z_{n+1}^{2}}}{\frac{z_{n+1} y_{n+1}}{y_{n+1}^{2}}}\right)=\left(\begin{array}{ccc}
E^{2}+W^{2} & 2 E & 1 \\
-E & -1 & 0 \\
1 & 0 & 0
\end{array}\right)\left(\begin{array}{c}
\overline{z_{n}^{2}} \\
\frac{z_{n} y_{n}}{y_{n}^{2}}
\end{array}\right)
$$

and suggesting their exponential behavior $\lambda^{n}$ with $\lambda=1+x$, obtains the equation for $x$

$$
x^{3}-x^{2}\left(E^{2}-4\right)-x\left(E^{2}-4\right)=W^{2}\left(2-3 x-x^{2}\right)
$$

Setting $E^{2}-4=4 \delta^{2}, W^{2}=4 \epsilon^{2} \delta^{2}$ and taking the limit $\delta \rightarrow 0, \epsilon \rightarrow 0, \delta / \epsilon^{2}=$ const, one can verify that (A.3) coincides with (44).

Analogously, for the fourth moments one has a system of equations

$$
\left(\begin{array}{c}
\frac{\overline{z_{n+1}^{4}}}{\frac{z_{n+1}^{3} y_{n+1}}{z_{n+1}^{2} y_{n+1}^{2}}} \\
\frac{z_{n+1} y_{n+1}^{3}}{y_{n+1}^{4}}
\end{array}\right)=\left(\begin{array}{ccccc}
E^{4}+6 E^{2} W^{2} & 4 E^{3}+12 E W^{2} & 6 E^{2}+6 W^{2} & 4 E & 1 \\
-E^{3}-3 E W^{2} & -3 E^{2}-3 W^{2} & -3 E & -1 & 0 \\
E^{2}+W^{2} & 2 E & 1 & 0 & 0 \\
-E & -1 & 0 & 0 & 0 \\
1 & 0 & 0 & 0 & 0
\end{array}\right)\left(\begin{array}{c}
\overline{z_{n}^{4}} \\
\frac{z_{n}^{3} y_{n}}{z_{n}^{2} y_{n}^{2}} \\
\frac{z_{n} y_{n}^{3}}{y_{n}^{4}}
\end{array}\right)
$$

Suggesting that the moments behave as $\lambda^{n}$ with $\lambda=1+x$, we have the equation for $x$

$$
\begin{gathered}
-x^{5}+x^{4}\left(E^{2}+1\right)\left(E^{2}-4\right)+x^{3}\left(E^{2}-4\right)\left(-E^{4}+5 E^{2}+1\right)-x^{2} 2 E^{2}\left(E^{2}-4\right)^{2}-x E^{2}\left(E^{2}-4\right)^{2}+ \\
+W^{2}\left[-6 E^{2}\left(E^{2}-4\right)-15 x E^{2}\left(E^{2}-4\right)+x^{2}\left(-12 E^{4}+60 E^{2}-6\right)+\right. \\
\left.+x^{3}\left(-3 E^{4}+30 E^{2}-9\right)+x^{4}\left(6 E^{2}-3\right)\right]=0
\end{gathered}
$$

where terms of the higher order in $W^{2}$ are omitted. Setting $E^{2}-4=4 \delta^{2}, W^{2}=4 \epsilon^{2} \delta^{2}$ and retaining the main terms at the indicated limiting transition, we come to Eq.48.

\footnotetext{
${ }^{7}$ In this case the parameter $\gamma \sim 1$ and does not have the essential evolution.
} 


\section{Appendix 2. The asymptotic forms of the integral (70).}

Using the evenness of the integrand of (70) in variables $\tilde{\varphi}=\varphi-\pi / 2, \tilde{\theta}=\theta-\pi / 2$ and setting $x=\sin \varphi$, $y=\sin \theta$, we have

$$
\begin{gathered}
P(\rho)=\frac{1}{\pi^{2} t} \int_{-1}^{1} \frac{d x}{\sqrt{1-x^{2}}} \int_{-1}^{1} \frac{d y}{\sqrt{1-y^{2}}} \exp \left\{-\frac{S(x, y)}{t}\right\} \\
S(x, y)=\left(\Delta_{1} \sqrt{1+\rho} x+\Delta_{2} \sqrt{\rho} y\right)^{2}+\rho\left(1-y^{2}\right)
\end{gathered}
$$

Configuration of saddle points is essentially different for $\rho<\rho_{c}$ and $\rho>\rho_{c}$, where $\rho_{c}=\Delta_{1}^{2}$. In the first case the maximum of the exponent is reached at $x=x_{c}, y=-1$ or $x=-x_{c}, y=1$, where $x_{c}=$ $\Delta_{2} \sqrt{\rho} / \Delta_{1} \sqrt{1+\rho}$, while in the second case at $x=1$, $y=-1$ or $x=-1, y=1$. Let $\delta x$ and $\delta y$ are deviations of $x$ and $y$ from the extremum point. For $\rho<\rho_{c}$ we retain in $S(x, y)$ the quadratic term in $\delta x$ and the linear term in $\delta y$, setting $x=x_{c}, 1-y^{2}=$ $2 \delta y$ in the pre-exponential; then

$$
P(\rho)=\frac{1}{\pi} \sqrt{\frac{1}{\rho\left(\rho_{c}-\rho\right)},}
$$

which is the limiting form of the distribution for $t \rightarrow$ 0 .

For $\rho>\rho_{c}$ we have

$S(x, y)=S_{c}+A \delta x+B \delta y+C\left(\delta x-x_{c} \delta y\right)^{2}-\rho(\delta y)^{2}$,

where $S_{c}, A, B$ are defined in Eq.73 and $C=\Delta_{1}^{2}(1+$ $\rho)$. For large $\rho$ the linear terms in $\delta x, \delta y$ are sufficient in $S(x, y)$ and in radicals; then

$$
P(\rho)=\frac{1}{\pi} \sqrt{\frac{1}{A B}} \exp \left(-\frac{S_{c}}{t}\right),
$$

which is the last asymptotics in (72), (74)-(77).

The results $(A .7),(A .9)$ are not applicable for $\rho$ close to $\rho_{c}$, since the coefficient $A$ turns to zero at $\rho=\rho_{c}$. Setting $\delta \tilde{x}=\delta x-x_{c} \delta y$ and omitting the last term in $(A .8)$, we have

$$
S(x, y)=S_{c}+A \delta \tilde{x}+C(\delta \tilde{x})^{2}+2 \rho \delta y .
$$

For $A^{2} \ll C t$ fluctuations of $\delta \tilde{x}$ are determined by the quadratic term, and $A \delta \tilde{x}$ can be omitted; then in the small vicinity of $\rho_{c}$

$$
P(\rho)=\frac{1}{2 \pi} \sqrt{\frac{1}{2 \pi \rho}} \frac{\Gamma(1 / 4)}{(4 C t)^{1 / 4}}, \quad\left|\rho-\rho_{c}\right| \lesssim(C t)^{1 / 2},
$$

so divergency at $\rho \rightarrow \rho_{c}$ is eliminated and the third asymptotics in (72) and (75) is recovered. Under the indicated condition, this result remains valid for $\rho<\rho_{c}$, when $A$ is negative, but small in modulus. In fact, Eq.(A.11) takes place in the cases $t \ll \Delta_{1}^{2} \ll 1$ and $t \ll 1 \ll \Delta_{1}^{2}$, when the fluctuation $\delta y \sim t / \rho$ is small in comparison with $\delta \tilde{x} \sim \sqrt{t / C}$, so $\delta \tilde{x} \approx \delta x$ and one can set $1-x^{2}=2 \delta \tilde{x}, 1-y^{2}=2 \delta y$ in the pre-exponential. The inverse situation is realized in the case $\Delta_{1}^{2} \gg 1, t \gg 1$, when $\delta y \gg \delta \tilde{x}$ and $1-x^{2} \approx 2 \delta x=2 \delta \tilde{x}+2 x_{c} \delta y \approx 2 \delta y$ in the preexponential; then we come to the result

$$
\begin{gathered}
P(\rho)=\frac{1}{2 \pi^{2}} \sqrt{\frac{\pi}{\rho \Delta_{1}^{2} t}} \ln \frac{\Delta_{1}^{2} t}{\rho} \exp \left(-\frac{S_{c}}{t}\right), \\
\rho_{c} / t \lesssim \rho \lesssim \rho_{c} t,
\end{gathered}
$$

determining the penultimate asymptotics in (76), (77). The condition $A^{2} \ll C t$ corresponds to $\left(\rho-\rho_{c}\right)^{2} \ll \Delta_{1}^{2} \rho t$, which for $t \gg 1$ is reduced to the given in $(A .12)$. If $(A .11)$ is valid in the small vicinity of $\rho_{c}$, the wide range of validity arises for (A.12).

For small $\rho$ the saddle point approximation is not applicable, and the initial form of (70) is convenient. If $t \ll \Delta_{1}^{2}$, then saddle point integration over $\varphi$ is still possible and leads to expression

$$
\begin{gathered}
P(\rho)=\frac{1}{\pi^{2} t} \int_{-\pi / 2}^{\pi / 2} d \theta \sqrt{\frac{\pi t}{\Delta_{1}^{2}-\rho+\Delta_{2}^{2} \rho \sin ^{2} \theta}} . \\
\cdot \exp \left\{-\frac{\rho}{t} \sin ^{2} \theta\right\} .
\end{gathered}
$$

For $\Delta_{1}^{2} \ll 1$ one has $\Delta_{2} \approx 1$; if $\rho \ll t$, then only $\Delta_{1}^{2}$ can be retained in the denominator, while integration over $\theta$ can be produced by expansion of the exponent

$$
P(\rho)=\frac{1}{\pi} \sqrt{\frac{\pi}{\Delta_{1}^{2} t}} \exp \left(-\frac{\rho}{2 t}\right), \quad \rho \ll t,
$$

which is the first result in $(72),(75),(76)$. For $\rho \gtrsim t$ one returns to the saddle point results $(A .7),(A .9)$, (A.10).

In the case $\Delta_{1}^{2} \ll 1$ and $t \gg \Delta_{1}^{2}$ the region of small $\rho$ is determined by the condition $\rho(1+\rho) \ll t^{2} / \Delta_{1}^{2}$ and the integral (70) is calculated by expansion over $S(\rho, \varphi, \theta) / t$ till the second order, which gives the first result in (74), (77); for the opposite inequality we have $A \approx B \approx 2 \Delta_{1} \Delta_{2} \sqrt{\rho(1+\rho)} \gg t$ and $A^{2} \gg C t$, which is sufficient for validity of $(A .9)$. 
In the case $\Delta_{1}^{2} \gg 1$ and $t \ll \Delta_{1}^{2}$ Eq.(A.13) remains valid, but its analysis is more complicated. For $t \ll 1$ we have the familiar situation: in the interval $\rho \lesssim t$ one can retain $\Delta_{1}^{2}$ in the denominator and obtain (A.14), while in the interval $\rho \gtrsim t$ the saddle point results $(A .7),(A .9),(A .10)$ are valid. For $1 \ll t \ll \Delta_{1}^{2}$ the result $(A .14)$ is valid only for $\rho \lesssim 1$. In the interval $1 \lesssim \rho \lesssim t$ the term $\Delta_{2}^{2} \rho \sin ^{2} \theta$ is dominated in the denominator of (A.13), while the quantity $\Delta_{1}^{2}-\rho$ is necessary only for cutoff of the logarithmic divergency:

$$
P(\rho)=\frac{1}{\pi^{2}} \sqrt{\frac{\pi}{\rho \Delta_{1}^{2} t}} \ln \frac{\Delta_{1}^{2} \rho}{\rho_{c}-\rho}, \quad 1 \lesssim \rho \lesssim t .
$$

In the interval $t \lesssim \rho \lesssim \rho_{c}$ the exponent restrict integration in $(A .13)$ by values $\theta^{2} \lesssim t / \rho$, so $\Delta_{1}^{2} \rho$ in (A.15) is changed by $\Delta_{1}^{2} t$. In fact, both results are actual only for $\rho \ll \rho_{c}$, since in the interval $\rho \gtrsim \rho_{c} / t$ divergency at $\rho \rightarrow \rho_{c}$ is eliminated due to nonlinear terms in $(A .8)$ and the result $(A .12)$ is valid; so we have $\ln \rho$ for $1 \lesssim \rho \lesssim t$ and $\ln t$ for $t \lesssim \rho \lesssim \rho_{c} / t$, as is reflected in (76).

In the case $\Delta_{1}^{2} \gg 1$ and $t \gg \Delta_{1}^{2}$, expansion over $S(\rho, \varphi, \theta) / t$ is possible in the interval $\rho \lesssim t / \Delta_{1}^{2}$ and leads to the first result (77). In the interval $\rho \gtrsim t / \Delta_{1}^{2}$ expression (A.13) is valid, where $\left|\Delta_{1}^{2}-\rho\right| \ll t$, $\Delta_{2}^{2} \rho \gg t$ and the latter term is dominant in the denominator; the logarithmic divergency is removed due to restriction $\left(\Delta_{2}^{2} \rho / t\right) \sin ^{2} \theta \gtrsim 1$, which is necessary for the saddle point integration over $\varphi$ and validity of (A.13). If $\rho \lesssim t$, then the exponent in (A.13) is not essential and the second result (77) holds. If $\rho \gtrsim t$, then we have the saddle point situation and validity of $(A .12)$ and (A.9).

\section{References}

[1] P. W. Anderson, D. J. Thouless, E. Abrahams, D. S. Fisher, Phys. Rev. B 22, 3519 (1980).

[2] R. Landauer, IBM J. Res. Dev. 1, 223 (1957); Phil. Mag. 21, 863 (1970); Z. Phys. 68, 217 (1987).

[3] E. N. Economou, C. M. Soukoulis, Phys. Rev. Lett. 46, 618 (1981).

[4] D. S. Fisher, P. A. Lee, Phys. Rev. B 23, 6851 (1981).

[5] D. C. Langreth, E. Abrahams, Phys. Rev. B 24, 2978 (1981).
[6] P. W. Anderson, Phys. Rev. B 23, 4828 (1981);

H. L. Engquist, P. W. Anderson, Phys. Rev. B 24, 1151 (1981).

[7] D. J. Thouless, Phys. Rev. Lett. 47, 972 (1981).

[8] M. Buttiker, Phys. Rev. Lett. 57, 1761 (1986). M. Buttiker, Y. Imry, R. Landauer, S. Pinhas, Phys. Rev. B 31, 6207 (1985).

[9] M. Ya. Azbel, J. Phys. C 14, L225 (1981).

[10] A. D. Stone, A. Szafer, IBM J. Res. Dev. 32, 384 (1988).

[11] I. M. Suslov, J. Exp. Theor. Phys. 115, 897 (2012) [Zh. Eksp. Teor. Fiz. 142, 1020 (2012)].

[12] I. M. Suslov, J. Exp. Theor. Phys. 124, 763 (2017) [Zh. Eksp. Teor. Fiz. 151, 897 (2017)].

[13] V. I. Melnikov, Sov. Phys. Sol. St. 23, 444 (1981).

[14] A. A. Abrikosov, Sol. St. Comm. 37, 997 (1981).

[15] N. Kumar, Phys. Rev. B 31, 5513 (1985).

[16] B. Shapiro, Phys. Rev. B 34, 4394 (1986).

[17] P. Mello, Phys. Rev. B 35, 1082 (1987).

[18] B. Shapiro, Phil. Mag. 56, 1031 (1987).

[19] I. M. Lifshitz, S. A. Gredeskul, L. A. Pastur, Introduction to the Theory of Disordered Systems, Nauka, Moscow, 1982.

[20] X. Chang, X. Ma, M. Yepez, A. Z. Genack, P. A. Mello, Phys. Rev. B 96, 180203 (2017).

[21] L. I. Deych, D. Zaslavsky, A. A. Lisyansky, Phys. Rev. Lett. 81, 5390 (1998).

[22] L. I. Deych, A. A. Lisyansky, B. L Altshuler, Phys. Rev. Lett. 84, 2678 (2000); Phys. Rev. B 64, 224202 (2001).

[23] L. I. Deych, M. V. Erementchouk, A. A. Lisyansky, Phys. Rev. Lett. 90, 126601 (2001).

[24] L. D. Landau, E. M. Lifshitz, Quantum Mechanics, Pergamon, 1977.

[25] B. L. Altshuler, JETP Lett. 41, 648 (1985) [Pis'ma Zh. Eksp. Teor. Fiz.41, 530 (1985)]; 
[26] P. A. Lee, A. D. Stone, Phys. Rev. Lett. 55, 1622 (1985).

[27] D. Braun et al, Phys. Rev. B 64, 155107 (2001).

[28] I. Travenec, Phys. Rev. B 69, 033094 (2004).

[29] M. Ruhlender, P. Markos, C. M. Soukoulis, Phys. Rev. B 64, 172202 (2001).

[30] A. O. Gel'fand, Calculus of Finite Differences, Nauka, Moscow, 1967.

[31] I. M. Suslov, J. Exp. Theor. Phys. 127, 131 (2018) [Zh. Eksp. Teor. Fiz. 154, 152 (2018)].

[32] M. Kappus, F. Wegner, Z. Phys. B 45, 15 (1981).

[33] V. E. Kravtsov, V. I. Yudson, Ann. Phys. (NY) 326, 1672 (2011).

[34] S. Washburn, R. A. Webb, Adv. Phys. 35, 375 (1986).

[35] D. Mailly, M. Sanquer, J. Phys. (France) I 2, 357 (1992).

[36] E. Abrahams, P. W. Anderson, D. C. Licciardello, and T. V. Ramakrishman, Phys. Rev. Lett. 42, 673 (1979).

[37] A. Cohen, Y. Roth, B. Shapiro, Phys. Rev. B 38, 12125 (1988).

[38] I. M. Suslov, Phil. Mag. 99, 247 (2019). 